February 1, 2008

LBNL-41198, UCB-PTH-97/68

hep-th/9801060

\title{
Branes and Dynamical Supersymmetry Breaking
}

\author{
Jan de Boer, Kentaro Hori, Hirosi Ooguri and Yaron Oz \\ Department of Physics, University of California at Berkeley \\ 366 Le Conte Hall, Berkeley, CA 94720-7300, U.S.A. \\ and \\ Theoretical Physics Group, Mail Stop 50A-5101 \\ Ernest Orlando Lawrence Berkeley National Laboratory \\ Berkeley, CA 94720, U.S.A.
}

\begin{abstract}
We study dynamical supersymmetry breaking in four dimensions using the fivebrane of $M$ theory, in particular for the Izawa-Yanagida-IntriligatorThomas (IYIT) model, which we realize as the worldvolume theory of a certain M-theory fivebrane configuration. From the brane point of view, supersymmetry is broken when a holomorphic configuration with the proper boundary conditions does not exist. We discuss the difference between explicit and spontaneous supersymmetry breaking and between runaway behavior and having a stable vacuum. As a preparation for the study of the IYIT model, we examine a realization of the orientifold four-plane in $M$ theory. We derive known as well as new results on the moduli spaces of $N=2$ and $N=1$ theories with symplectic gauge groups. These results are based on a hypothesis that a certain intersection of the fivebrane and the $\mathbf{Z}_{2}$ fixed plane breaks supersymmetry. In the IYIT model, we show that the brane exhibits runaway behavior when the flavor group is gauged. On the other hand, if the flavor group is not gauged, we find that the brane does not run away. We suggest that a stable supersymmetry-breaking vacuum is realized in the region beyond the reach of the supergravity approximation.
\end{abstract}




\section{Introduction}

One of the most important and interesting issues in supersymmetric gauge theories is the dynamical breaking of supersymmetry. Under what conditions and by what mechanisms is supersymmetry dynamically broken? If supersymmetry is broken, is there a non-supersymmetric stable vacuum? If there is, what is the vacuum energy? There have been a lot of important works from various point of view concerning such questions but they still remain as difficult and fascinating as before.

If there is a new method for analyzing field theory, it is worth examining whether it sheds a new light on the issue of dynamical supersymmetry breaking. The study of the worldvolume dynamics of branes in string theory and $M$ theory has given a new perspective on the study of the strong coupling dynamics of supersymmetric gauge theories in various dimensions [1-48]. The worldvolume theory depends on parameters such as the string coupling constant which are absent in the ordinary gauge theory. For generic values of such parameters, it is different from the gauge theory since it interacts with the bulk degrees of freedom in the ten or eleven-dimensional space-time. In addition, it contains

Kaluza-Klein modes associated with the compactification of the worldvolume theory to lower dimensions. However, there are certain quantities which are independent of such differences, and one can obtain exact results by going to the region of the parameter space where the worldvolume dynamics simplifies, e.g., the eleven-dimensional supergravity limit of $M$ theory. Examples of such quantities are holomorphic or BPS objects such as the effective holomorphic gauge coupling constant $[6,15,16]$, vevs of some chiral operators $[18,19,21,30,36]$, and the mass or tension of BPS states [19, 26, 32, 41,39]. Furthermore, when the universality class is expected to remain the same, we can make predictions about qualitative features of the theory which might be harder to obtain from conventional field theory methods [19]. Therefore, it is interesting to see whether branes can provide a new point of view on dynamical supersymmetry breaking.

In this paper, we construct $M$ theory fivebrane realizations of four-dimensional gauge theories that exhibit dynamical supersymmetry breaking. Some of them are expected to have a stable non-supersymmetric vacuum, the others are not. We study how the existence or absence of a stable vacuum is realized in the fivebrane picture. A field theory model of dynamical supersymmetry breaking without chiral matter and with a stable nonsupersymmetric vacuum was constructed recently by Izawa-Yanagida [49] and IntriligatorThomas [50] using knowledge of the strong coupling dynamics of supersymmetric gauge theory with symplectic gauge group [51,52]. Their model (which we shall call the IYIT model) exhibits dynamical supersymmetry breaking and can be argued to have a stable 
non-supersymmetric vacuum by computing the one-loop correction to the scalar potential. If we gauge the orthogonal flavor group, supersymmetry is still broken but the same oneloop computation exhibits runaway behavior and does not show the existence of a stable vacuum. Using the brane picture, we will present evidence that such a vacuum does in fact not exist. On the other hand, if the flavor group is not gauged, we find that the brane does not run away. We suggest that a stable vacuum without supersymmetry, if it exists, is realized in the region beyond the reach of the supergravity approximation. The situation is very similar to what happens in the field theory analysis of dynamical supersymmetry breaking of this type. There, one has to make an assumption about the behavior of the Kähler potential in the strong coupling region of the gauge theory in order to prove the existence of a stable vacuum which does break supersymmetry.

Supersymmetric gauge theories with symplectic gauge group can be realized as the worldvolume dynamics of Type IIA branes by introducing an orientifold. To realize the theories mentioned above, we chose to introduce an orientifold four-plane. Although we do not have a good understanding of the dynamics of fivebranes in $M$ theory in the presence of a Type IIA orientifold four-plane, we can show that the brane reproduces information on the moduli space of supersymmetric vacua by introducing a simple hypothesis concerning the intersection of the fivebrane and the $\mathbf{Z}_{2}$ fixed plane. The hypothesis indicates the presence of a force (which can be either finite or infinite) between the points of intersection, which plays a crucial role when we discuss the issue of existence of a stable non-supersymmetric vacuum.

The paper is organized as follows. In section 2, we present the general idea on spontaneous supersymmetry breaking in terms of branes. In particular, we clarify the distinction between spontaneous and explicit breaking and demonstrate the concepts using a toy model.

In section 3, we examine the simple hypothesis mentioned above by studying the brane configurations corresponding to $N=2$ and $N=1$ supersymmetric QCD with symplectic gauge group. We find that the brane picture together with the hypothesis correctly reproduce the known results of $N=2$ and $N=1$ field theories and yield a new result about the total moduli space of vacua of $N=2$ theories. Part of the results of this section were recently obtained in $[44,35]$.

In section 4, we study the IYIT model in the brane framework. We first determine the structure of supersymmetric vacua of the model with some perturbations using ordinary field theory methods. Next, we realize the perturbed system via branes, using the electricmagnetic duality as a guide. Then, we consider the system with supersymmetry breaking 
and discuss the existence of a stable non-supersymmetric vacuum. Under the plausible assumption that the potential energy between a fivebrane and the orientifold is negligible when the distance between the fivebrane and the orientifold is much larger than the eleven dimensional Planck length, we show that the brane does not run away.

In section 5, we study the IYIT model with the orthogonal flavor group gauged. We examine the space of supersymmetric vacua for general numbers of colors and flavors using the brane based on the basic hypothesis, and find that the brane reproduces the correct field theory results. For the number of colors and flavors corresponding to the IYIT model, we will present evidence that there is runaway behavior.

\section{Branes and Supersymmetry Breaking}

In this section we will discuss the general aspects of supersymmetry breaking in the brane framework, and present a simple two dimensional model as an illustration.

\subsection{General Idea}

Supersymmetric gauge theories can be studied in various dimensions by realizing them as theories on the world volume of branes. Of particular importance to us in the study of supersymmetry breaking will be $N=1$ supersymmetric gauge theories in four dimensions. These are constructed using configurations of intersecting branes in Type IIA string theory which capture the semiclassical features of the gauge theories. In order to study the quantum properties we have to lift the brane configuration to $M$ theory [6]. It is described by a fivebrane wrapping a Riemann surface holomorphically embedded in the space-time (or more precisely, in a Calabi-Yau three-fold which is a part of the eleven-dimensional space-time). The Riemann surface encodes information about the supersymmetric ground states of the theory; For example, the effective gauge coupling constant in an abelian Coulomb phase $[6,15,16,43]$, chiral symmetry breaking, and gaugino or monopole condensation in a confining phase $[18,19,21,30]$, vevs of some chiral operators such as mesons and baryons in a Higgs phase [18, 21,36, 38, 44, 35], and the mass or tension of BPS states [26, 32, 41,39].

The Riemann surface is a supersymmetric cycle [62-64] and the fivebrane wrapping the Riemann surface is a supersymmetric configuration, namely a BPS object. The amount of supersymmetry preserved by the fivebrane configuration depends on the details of the Riemann surface. The signal for supersymmetry breaking is that the fivebrane is no longer wrapping a holomorphic curve but rather a nonholomorphic real two dimensional surface. 
Such a fivebrane configuration is not a BPS object because it breaks all supersymmetry completely. Therefore the issue of the stability of the configuration arises.

Generically we can have a stable configuration if the two dimensional surface is of minimal area with respect to the space-time metric. There are however some subtleties that will arise. The first subtlety is associated with the fact that the brane configuration can be a factorized surface. In such a case the disconnected components affect each other via the gravitational force which has to be taken into account when analyzing the stability of the configuration. This effect was crucial for instance in deriving Higgs branch metrics in [43]. The second subtlety is associated with the orientifold four-plane which will be used in this paper in order to get symplectic gauge groups. The orientifold creates a force that acts on the fivebrane intersecting with the $\mathbf{Z}_{2}$ fixed plane. Taking such an effect into account was actually necessary in order to obtain the hyperelliptic curves describing the $N=2$ Coulomb branches of gauge theories based on symplectic and orthogonal gauge groups [15]. We will see in section 3 that this is also required in order for $N=1$ fivebrane configurations to correctly describe the supersymmetric ground states of the gauge field theories. Clearly this effect should also be taken into account when studying the stability of the non-supersymmetric fivebrane configurations.

The holomorphic fivebrane configurations are of minimal volume in their homology class. They correspond to zero energy vacua of the field theory. The non-holomorphic configurations have a larger volume. In the absence of the forces that we discussed above the difference between the volumes of the non-holomorphic and holomorphic configurations corresponds to the non-zero energy of the non-supersymmetric vacuum. When the above forces are relevant their contribution to the potential energy has to be taken into account.

There is a difference between explicit and spontaneous supersymmetry breaking. Although in both cases the two dimensional surface is not holomorphic there is a difference between the asymptotic boundary conditions at infinity of the surface.

The fivebrane we are considering is wrapped on a two-dimensional surface which is non-compact in one direction. In order to realize a four-dimensional gauge theory from the six-dimensional worldvolume theory, we must restrict the allowed motion of the brane by specifying a set of boundary conditions at infinity. In general, a symmetry of the original theory is also a symmetry of the restricted system when it preserves the boundary condition at infinity, whereas it is explicitly or anomalously broken when it breaks the boundary condition. When a symmetry remains, it is unbroken by a choice of vacuum if it completely preserves the corresponding fivebrane configuration, but it is spontaneously 
broken otherwise. For an example of spontaneous breaking, consider the breaking $\mathbf{Z}_{2 n} \rightarrow$ $\mathbf{Z}_{2}$ of the discrete chiral symmetry of $N=1$ supersymmetric Yang-Mills theory by a choice of vacuum configuration $[18,19]$, or of the $N=2$ supersymmetric $\mathbf{C P}^{n-1}$ sigma model in two dimensions [65]. When a symmetry is spontaneously broken there is a degeneracy of vacuum configurations, as the example of $\mathbf{Z}_{2 n} \rightarrow \mathbf{Z}_{2}$ exhibits (there are $n$ distinct vacuum configurations).

As is well-known, the system of a flat and unrestricted fivebrane has the six-dimensional $(2,0)$ supersymmetry. If the action of any of the supersymmetry generators changes the boundary condition at infinity, the restricted system corresponding to the fourdimensional theory no longer has that particular supersymmetry, namely, that supersymmetry is absent from the start. This is the case when the asymptotic boundary condition is not holomorphic, and corresponds to an explicit supersymmetry breaking. Examples of this kind were given in the last section of [19]. If the action of some of the supersymmetry generators preserves the boundary condition at infinity, the restricted system is supersymmetric under the corresponding subalgebra. This is the case when the asymptotic boundary condition is holomorphic, and the supersymmetric four-dimensional theory may or may not have a supersymmetric ground state depending on whether or not there is an everywhere holomorphic curve obeying the boundary condition. If the supersymmetry is spontaneously broken, there must be massless fermions (Goldstone fermions). The action of the supercharges on a non-holomorphic minimal surface generates fermionic zero modes on the brane. When the asymptotic boundary condition is holomorphic, these decay at infinity of the surface and therefore correspond to fields in the four-dimensional theory. These can be identified with the Goldstone fermions associated with the spontaneous supersymmetry breaking.

To summarize the main points of the above discussion: A supersymmetric vacuum corresponds to a fivebrane wrapping a holomorphic complex curve. Breaking the supersymmetry of the gauge theory corresponds in the brane picture to the non-existence of a holomorphic curve describing the brane configuration. A non-supersymmetric stable vacuum corresponds to a fivebrane wrapping a nonholomorphic real two dimensional surface with minimal area, taking into account the force between the surface and the orientifold and the gravitational force between the possible different components of the surface. If supersymmetry is broken spontaneously the real two dimensional surface has asymptotic boundary conditions which are holomorphic. 


\subsection{Example: A Two-Dimensional Toy Model}

In the following we will present a two dimensional toy model for supersymmetry breaking that will illustrate some of the above discussion. We will consider a $U(1) N=2$ supersymmetric gauge theory in two dimensions (four supercharges) with one charged hypermultiplet. The theory can be obtained by dimensional reduction of an $N=1$ supersymmetric gauge theory in four dimensions. The vector multiplet of the theory contains as bosonic degrees of freedom the gauge field $A_{\mu}$ and a complex scalar $\sigma$ which arises from the $x^{2}, x^{3}$-components of the gauge field in the process of the dimensional reduction. The hypermultiplet contains as bosonic degrees of freedom two complex scalars that we will denote by $Q, \tilde{Q}$.

We can introduce a Fayet-Iliopoulos (FI) D-term, $-r \int d^{2} x \mathrm{~d}^{4} \theta V$, where $r$ is a real FI parameter and $V$ is the $U(1)$ vector superfield. In addition, we introduce a complex mass term $\int d^{2} x \mathrm{~d}^{2} \theta m Q \tilde{Q}+$ c.c.. The total scalar potential $U$ of the theory reads

$$
2 e^{2} U=|\sigma Q|^{2}+|\sigma \tilde{Q}|^{2}+|m|^{2}\left(|Q|^{2}+|\tilde{Q}|^{2}\right)+\left(|Q|^{2}-|\tilde{Q}|^{2}-e^{2} r\right)^{2}
$$

where $e$ is the two-dimensional gauge coupling constant. When $r=Q=\tilde{Q}=0$ the potential energy is zero and there is a complex one dimensional moduli space of supersymmetric vacua parametrized by $\sigma$. For $m=0, r \neq 0$ there is a complex one dimensional Higgs branch. Finally, if both $m$ and $r$ are nonzero, the potential energy is always nonzero and supersymmetry is spontaneously broken. If $2 e^{2} r \leq-|m|^{2}$, the minimum of the potential is at $\left(|Q|^{2},|\tilde{Q}|^{2}\right)=\left(0,-e^{2} r-|m|^{2} / 2\right)$, for $-|m|^{2} \leq 2 e^{2} r \leq|m|^{2}$ it is at $\left(|Q|^{2},|\tilde{Q}|^{2}\right)=(0,0)$, and for $|m|^{2} \leq 2 e^{2} r$ it is at $\left(|Q|^{2},|\tilde{Q}|^{2}\right)=\left(e^{2} r-|m|^{2} / 2,0\right)$. In the second case there is a family of non-supersymmetric vacua parametrized by $\sigma$.

Brane configurations of $N=2$ gauge theories in two dimensions have been studied in [65]. In order to realize the above $U(1)$ gauge theory we consider the following brane configuration in type IIA string theory. An NS fivebrane with worldvolume coordinates $\left(x^{0}, x^{1}, x^{2}, x^{3}, x^{4}, x^{5}\right)$, a NS' fivebrane with worldvolume coordinates $\left(x^{0}, x^{1}, x^{2}, x^{3}, x^{8}, x^{9}\right)$, a D4 brane with worldvolume coordinates $\left(x^{0}, x^{1}, x^{7}, x^{8}, x^{9}\right)$ located between the two fivebranes, and a D2 brane with worldvolume coordinates $\left(x^{0}, x^{1}, x^{6}\right)$ stretched between the NS and NS' branes in the $x^{6}$ direction. The theory on the worldvolume of the D2 brane in $\left(x^{0}, x^{1}\right)$ has $N=2$ supersymmetry, gauge group $U(1)$ and one hypermultiplet charged under the $U(1)$. This configuration is T-dual to the $N=1$ four dimensional configuration of [3].

The gauge coupling of the theory is related to the distance between the NS and $\mathrm{NS}^{\prime}$ branes in the $x^{6}$ direction, $1 / e^{2}=\left(\ell_{s t} / g_{s t}\right) \Delta x^{6}$. The FI parameter of the theory is related 
to the distance between the NS and $\mathrm{NS}^{\prime}$ branes in the $x^{7}$ direction, $-r=\left(1 / \ell_{s t} g_{s t}\right) \Delta x^{7}$. The value of the coordinates $x^{2}+i x^{3}$ of the $\mathrm{D} 2$ brane is related to $\sigma$ by $x^{2}+i x^{3}=\ell_{s t}{ }^{2} \sigma$ and the complex mass is related to the $x^{4}, x^{5}$ position of the $D 4$ brane via $m \ell_{s t}{ }^{2}=x^{4}+i x^{5}$.

When the distance between the NS and $\mathrm{NS}^{\prime}$ branes in the $x^{7}$ direction is zero the brane configuration preserves four supercharges and for each value of the coordinates $x^{2}+i x^{3}$ of the D2 brane it corresponds to a supersymmetric vacuum. Similarly, by breaking the D2 brane on the D4 brane we see supersymmetric vacua for $r \neq 0, m=0$.

Consider now the most general case $r \neq 0$ and $m \neq 0$. There are two possible configurations for the D2 brane. In what we will call configuration A, the D2 brane breaks on the D4 brane, while in what we will call configuration B it connects directly the NS and NS' branes without intersecting the D4 brane. In either case the D2 brane no longer has its worldvolume in the $\left(x^{0}, x^{1}, x^{6}\right)$ direction and therefore the brane configuration breaks supersymmetry. The vacua correspond to the configurations of minimal length, which is the two dimensional analog of the minimal area condition that we discussed in the four dimensional context. For configuration A, the length of the D2 brane equals

$$
L_{A}=\frac{g_{s t}}{\ell_{s t} e_{1}^{2}}+\sqrt{\left|m \ell_{s t}{ }^{2}\right|^{2}+\left(\frac{g_{s t}}{\ell_{s t} e_{2}^{2}}\right)^{2}}
$$

while for configuration B it equals

$$
L_{B}=\sqrt{\left(\frac{g_{s t}}{\ell_{s t} e^{2}}\right)^{2}+\left(r g_{s t} \ell_{s t}\right)^{2}} .
$$

Here, we introduced $\frac{1}{e_{1}^{2}}=\frac{\ell_{s t}}{g_{s t}}\left(x^{6}(D 4)-x^{6}(N S)\right)$ and $\frac{1}{e_{2}^{2}}=\frac{\ell_{s t}}{g_{s t}}\left(x^{6}\left(N S^{\prime}\right)-x^{6}(D 4)\right)$. For large $m$, the shortest brane configuration is configuration $\mathrm{B}$, corresponding to the field theory vacua with $|Q|=|\tilde{Q}|=0$. For large $r$, the shortest brane configuration is configuration $\mathrm{A}$, corresponding to the field theory vacua with $|Q| \neq 0$ or $|\tilde{Q}| \neq 0$. There is qualitative but no quantitative agreement with field theory, but this is what we expect since the brane configurations are not BPS [43]. This is already obvious by noticing that $L_{A}$ depends on $e_{1}^{2}$ and $e_{2}^{2}$ which do not correspond to parameters of field theory. Furthermore, we neglected the interaction between the two D2-brane components in configuration A. In general, for non-BPS configurations, there is no reason for this interaction to vanish. We expect that this additional interaction will lift the degeneracy of configuration A (corresponding to moving the component of the D2 brane between the D4 and NS' branes in the 8,9-direction), which has no counterpart in field theory.

On the other hand, $L_{B}$ does not depend on the parameters $e_{1}^{2}$ and $e_{2}^{2}$ and one can indeed quantitatively compute the energy density of the vacua with $|Q|=|\tilde{Q}|=0$ from the brane 
configuration. The energy density of these vacua is given by the difference between $L_{B}$ and the length $L_{0}=g_{s t} / \ell_{s t} e^{2}$ of the the supersymmetric brane configuration, multiplied by the membrane tension $1 / \ell_{11}{ }^{3}=1 / g_{s t} \ell_{s t}{ }^{3}$. This yields $\left(e^{2} r^{2} / 2\right)\left(1+o\left(\left(e^{2} r \ell_{s t}{ }^{2}\right)^{2}\right)\right)$, which converges to the field theory result $U=e^{2} r^{2} / 2$ in the limit $\ell_{s t} \rightarrow 0$ where the the string oscillation modes decouple.

In the non-supersymmetric brane configurations, the D2 brane is stretched between the NS, D4 and the NS' branes which by themselves preserve supersymmetry so that the boundary conditions on the minimal length line are supersymmetric. This is the geometrical manifestation of the fact that supersymmetry is broken spontaneously and not explicitly.

In order to study the quantum properties of the system we have to lift the brane configuration to $\mathrm{M}$ theory. The $\mathrm{D} 2$ brane becomes a membrane of $\mathrm{M}$ theory stretched between pairs of fivebranes. In this framework, the theta angle $\theta$ is seen as the distance between NS and NS' fivebrane in the $x^{10}$ direction [65] and the vacuum energy for configuration $\mathrm{B}$ becomes $e^{2}\left|i r+\frac{\tilde{\theta}}{2 \pi}\right|^{2} / 2$ where $\tilde{\theta}$ is the minimum among $\left|\Delta x^{10}+2 \pi n\right|, n \in \mathbf{Z}$. In particular, there is a discontinuity in the $\theta$-derivative of the vacuum energy which is also what we know in field theory.

\section{$3 \quad M$ Theory Description of Orientifold via $S p\left(N_{c}\right)$ Gauge Dy- namics}

In this section, we study properties of the fivebrane in $M$ theory in a geometry which is (locally) of the type $\mathbf{R}^{5} \times S^{1} \times \mathbf{R}^{5} / \mathbf{Z}_{2}$. We construct fivebrane configurations whose worldvolume dynamics describes some supersymmetric $\operatorname{Sp}\left(N_{c}\right)$ gauge theories and study the properties of the fivebrane by comparing with the structure of vacua of the corresponding gauge theories. ${ }^{1}$

In subsection 3.1, we study the vacuum structure of supersymmetric $S p\left(N_{c}\right)$ gauge theories, including $N=2$ SUSY QCD, $N=2$ broken to $N=1$ by a mass term for the adjoint, and $N=1$ SUSY QCD. The analysis is almost the same as that for gauge group $S U\left(N_{c}\right)$ gauge group given in [18], but there is an important difference: In the $N=2$ $S p\left(N_{c}\right)$ theory, the way the Higgs branch emanates from the quantum Coulomb branch has not been determined by a field theory argument so far. There is a related puzzle in

\footnotetext{
${ }^{1}$ This section is meant to be a preparation for the study of the brane realization of a model of supersymmetry breaking with a stable non-SUSY vacuum. Those who are mostly interested in the issue of dynamical supersymmetry breaking can skip this section except for the introductory part.
} 
the theory with finite adjoint mass, where we find an apparent discrepancy between the dimensions of the Higgs branches found in the $N=2$ and $N=1$ descriptions of the theory.

After reviewing some general properties of $M$ theory on $\mathbf{R}^{6} \times \mathbf{R}^{5} / \mathbf{Z}_{2}$ in subsection 3.2 , we construct in 3.3 fivebrane configurations whose worldvolume dynamics realizes the $S p\left(N_{c}\right)$ gauge theories based on the following hypothesis.

Let us denote by " $t$-configuration " a configuration that looks locally like a single fivebrane transversely intersecting the $\mathbf{Z}_{2}$ fixed plane at one point. More precisely, the six-dimensional worldvolume of the fivebrane shares $\mathbf{R}^{4}$ with the $\mathbf{Z}_{2}$ fixed plane $\mathbf{R}^{6}$ but the remaining two-dimensional part intersects the $\mathbf{Z}_{2}$ fixed plane transversally at one point. Then, the basic hypothesis is

t-configuration is not supersymmetric.

In particular, when there are (locally) two fivebranes intersecting the $\mathbf{Z}_{2}$ fixed plane at different points, the two points either attract or repel each other, corresponding to the two allowed choices of orientifold plane in the type IIA picture. When there is a Kaluza-Klein monopole, we will need a modified version of the hypothesis.

Based on such a hypothesis, we will find that the brane gives the correct field theory results on the structure of the moduli space of supersymmetric vacua. Actually, this hypothesis was used in the construction [15] of the Seiberg-Witten curve for the $N=2$ theory with symplectic gauge group. Here, we will see that this hypothesis is also crucial for reproducing other aspects of $N=2$ theories as well as properties of $N=1$ theories. Among other things, the hypothesis plays an essential role in reproducing the quantum modification of the classical constraint on the meson matrix in $N=1 \mathrm{SQCD}$ with $N_{f}=$ $N_{c}+1$. Moreover, based on this hypothesis, we can determine the way the Higgs branches are emanating from the quantum Coulomb branch in the $N=2$ theory, solving the puzzle about the theory with finite adjoint mass mentioned above.

\subsection{Supersymmetric $S p\left(N_{c}\right)$ Gauge Theories}

$N=2 S Q C D$

We start with describing facts about $N=2$ supersymmetric $\operatorname{Sp}\left(N_{c}\right)$ gauge theory with $N_{f}$ fundamental hypermultiplets. In $N=1$ language, it is an $S p\left(N_{c}\right)$ gauge theory with an adjoint chiral multiplet $\Phi_{b}^{a}$ and fundamental chiral multiplets $Q_{a}^{i}$. Here $a, b, \ldots=$ $1, \ldots, 2 N_{c}$ and $i, j, \ldots=1, \ldots, 2 N_{f}$ are the color and flavor indices. The matrix $J^{a b} \Phi_{b}^{c}$ is 
symmetric with respect to $a, c$ where $J^{a b}$ is the $S p\left(N_{c}\right)$ invariant skew-symmetric form. ${ }^{1}$ The superpotential of the theory is

$$
W=\sqrt{2} Q_{a}^{i} J^{a b} \Phi_{b}^{c} Q_{c}^{i}
$$

We will consider the case $N_{f}<2\left(N_{c}+1\right)$ where the theory is asymptotically free and generates a dynamical scale $\Lambda_{N=2}$. The classical $U(1)$ R-symmetry group (under which $\Phi$ carries charge 2) is broken by an anomaly to its discrete subgroup $\mathbf{Z}_{4\left(N_{c}+1\right)-2 N_{f}}$ while the $S U(2)$ R-symmetry remains exact. The flavor symmetry group is $S O\left(2 N_{f}\right)$.

The moduli space of vacua consists of Coulomb and Higgs branches. The Coulomb branch, where the gauge group is broken (generically) to $U(1)^{N_{c}}$, is parametrized by the $N_{c}$ Casimirs of $\Phi$ and is corrected by one-loop and instantons. The quantum Coulomb branch is described by the Seiberg-Witten curve of the form [54]

$$
y^{2}=x \prod_{a=1}^{N_{c}}\left(x-\phi_{a}^{2}\right)^{2}-\Lambda_{N=2}^{4 N_{c}+4-2 N_{f}} x^{N_{f}-1}
$$

which determines the effective gauge coupling and the Kähler metric. In the semi-classical region $\|\Phi\| \gg \Lambda_{N=2}, \pm \phi_{a}$ are interpreted as the eigenvalues of $\Phi$.

The (mixed Coulomb-)Higgs branches of the theory were analyzed in [55]. They are classified by an integer $r=1, \ldots,\left[N_{f} / 2\right]$. The $r$-th Higgs branch has quaternionic dimension $2 r N_{f}-\left(2 r^{2}+r\right)$ and emanates from a $N_{c}-r$ dimensional complex subspace of the Coulomb branch where there is an unbroken gauge group $S p(r)$. The Higgs branches themselves are not corrected by quantum effects, but the way they emanate from the Coulomb branch is. Under the naïve interpretation of $\pm \phi_{a}$ as the eigenvalues of $\Phi$, the $r$-th Higgs branch emanates from the locus where $r$ of the $\phi_{a}$ vanish. This is true for the values of $r$ where the $S p(r)$ gauge theory with $N_{f}$ flavors is not asymptotic free, i.e., for $r \leq\left[\left(N_{f}-2\right) / 2\right]$ (all but $r=\left[N_{f} / 2\right]$ ). For the case $r=\left[N_{f} / 2\right]$, however, since this low energy theory is asymptotically free and may be affected by strong dynamics, it is not clear whether the Higgs branch emanates from this locus. This problem was not solved in [55]. We will see that the brane gives the solution; it is true for $r=\left(N_{f}-1\right) / 2\left(N_{f}\right.$ odd), but is modified for $r=N_{f} / 2\left(N_{f}\right.$ even).

\footnotetext{
${ }^{1}$ In the symplectic basis, it is represented as the matrix $\left(J^{a b}\right)=\mathbf{1}_{N_{c}} \otimes\left(\begin{array}{cc}0 & -1 \\ 1 & 0\end{array}\right)$ where $\mathbf{1}_{N_{c}}$ is the
} unit matrix of size $N_{c}$. 
$N=2$ Broken to $N=1$

Let us give a bare mass $\mu$ to the adjoint chiral multiplet, breaking $N=2$ to $N=1$ supersymmetry

$$
W=\sqrt{2} Q_{a}^{i} J^{a b} \Phi_{b}^{c} Q_{c}^{i}+\mu \Phi_{b}^{a} \Phi_{a}^{b} .
$$

The $U(1)$ R-symmetry modified so that $\Phi$ and $Q Q$ both carry charge 1 is anomalously broken to $\mathbf{Z}_{2 N_{c}+2-N_{f}}$. For small values of $\mu$, we can use the description in terms of the Seiberg-Witten curve to analyze the structure of vacua. Most of the Coulomb branch is lifted except the discrete set of points where all the $\alpha$-cycles of the curve degenerate. As analyzed in [55], the remaining vacua are in the locus where $r=\left[\left(N_{f}-1\right) / 2\right]$ of the parameters $\phi_{a}$ in (3.2) vanish as well as in the locus where $r=N_{f}-N_{c}-2$ of them vanish. We shall call the former the $\mathbf{A}$ branch root and the latter the $\mathbf{B}$ branch root, ${ }^{2}$ and denote the corresponding Higgs branches by the $\mathbf{A}$ and $\mathbf{B}$ branch respectively. The $\mathbf{B}$ branch root is a single point which is invariant under $\mathbf{Z}_{2 N_{c}+2-N_{f}}$, whereas the $\mathbf{A}$ branch root consists of $2 N_{c}+2-N_{f}$ points, namely, the A branch consists of $2 N_{c}+2-N_{f}$ connected components; for $N_{f}$ odd these are related by $\mathbf{Z}_{2 N_{c}+2-N_{f}}$, but for $N_{f}$ even they fall into two separate orbits of $\mathbf{Z}_{2 N_{c}+2-N_{f}}$, each having $N_{c}+1-N_{f} / 2$ components. Pure Yang-Mills theory $\left(N_{f}=0\right)$ is an exceptional case where $N_{c}+1$ points related by the discrete R-symmetry remain supersymmetric.

For values of $\mu$ beyond $\Lambda_{N=2}$, the gauge coupling runs below the energy scale $\mu$ as in $N=1$ supersymmetric QCD whose dynamical scale $\Lambda_{N=1}$ is given by

$$
\Lambda_{N=1}^{3\left(N_{c}+1\right)-N_{f}}=\mu^{N_{c}+1} \Lambda_{N=2}^{2\left(N_{c}+1\right)-N_{f}} .
$$

If $\mu \gg \Lambda_{N=1}$, we can integrate out the heavy field $\Phi$. Then, the theory at energies below $\mu$ can be considered as $N=1$ SQCD with tree level superpotential

$$
\Delta W=-\frac{1}{2 \mu} \operatorname{Tr}\left(M^{2}\right),
$$

where $M^{i j}=Q^{i} Q^{j}=J^{a b} Q_{a}^{i} Q_{b}^{j}$ is the meson matrix whose components form a basis of gauge invariant chiral superfields. The superpotential $\Delta W$ breaks the flavor symmetry $S U\left(2 N_{f}\right)$ of $N=1 \mathrm{SQCD}$ to $S O\left(2 N_{f}\right)$. In the following, we describe the structure of vacua of this theory.

\footnotetext{
${ }^{2}$ In [55], only the $\mathbf{B}$ branch (present only for $N_{f}>N_{c}+1$ ) is identified. But this cannot be the whole thing, as is evident by considering the case $N_{c}=1(S p(1)=S U(2)), N_{f}=2$ where two points in the Coulomb branch remain. Actually, only degenerations of the form $y^{2}=x[\cdots]^{2}$ are considered in [55]. The $\mathbf{A}$ branch root corresponds to degenerations of the form $y^{2}=(x-a)[\cdots]^{2}, a \neq 0$. We thank A. Shapere for a discussion on this point. This was also recently noticed in [44].
} 
The pure $N=1$ super-Yang-Mills theory $\left(N_{f}=0\right)$ has $N_{c}+1$ massive vacua with gaugino condensation which breaks the discrete chiral symmetry as $\mathbf{Z}_{2\left(N_{c}+1\right)} \rightarrow \mathbf{Z}_{2}$. These correspond to the $N_{c}+1$ completely degenerate curves found in the $N=2$ analysis which are related by the R-symmetry action.

For $0<N_{f}<N_{c}+1$, an Affleck-Dine-Seiberg type superpotential is generated in the $N=1 S p\left(N_{c}\right)$ SQCD [52]. By a holomorphy argument as in $[56,18]$, we can show that the exact effective superpotential is obtained by adding to $\Delta W$ the term

$$
W_{\text {eff }}=\left(N_{c}+1-N_{f}\right)\left(\frac{\Lambda_{N=1}^{3\left(N_{c}+1\right)-N_{f}}}{\operatorname{Pf} M}\right)^{1 /\left(N_{c}+1-N_{f}\right)}-\frac{1}{2 \mu} \operatorname{Tr}\left(M^{2}\right) .
$$

For $N_{f}=N_{c}+1$, the moduli space of vacua of $N=1 \mathrm{SQCD}$ is modified by quantum corrections and is given by $\operatorname{Pf} M=\Lambda_{N=1}^{2\left(N_{c}+1\right)}$ [52]. We therefore expect that the effective superpotential of our model is given by

$$
W_{\text {eff }}=X\left(\operatorname{Pf} M-\Lambda_{N=1}^{2 N_{c}+2}\right)-\frac{1}{2 \mu} \operatorname{Tr}\left(M^{2}\right),
$$

where $X$ is a chiral superfield playing the role of a Lagrange multiplier.

For $N_{f}=N_{c}+2$, the exact superpotential of $N=1 \mathrm{SQCD}$ is given by $W=$ $-\operatorname{Pf} M / \Lambda^{2 N_{c}+1}$ [52]. The effective superpotential of our model is expected to be

$$
W_{\text {eff }}=-\frac{\operatorname{Pf} M}{\Lambda_{N=1}^{2 N_{c}+1}}-\frac{1}{2 \mu} \operatorname{Tr}\left(M^{2}\right) .
$$

For $N_{f}>N_{c}+2$, there is another theory whose low energy physics is equivalent to the one of the $N=1 \mathrm{SQCD}$. This dual magnetic theory is an $\operatorname{Sp}\left(\widetilde{N}_{c}=N_{f}-N_{c}-2\right)$ gauge theory with $2 N_{f}$ fundamental chiral multiplets $q_{i}^{a}$ and a gauge singlet chiral multiplet $M$ (which is identified with the meson field $Q Q$ of the electric theory) which has a tree level superpotential $W_{\text {mag }}=\frac{1}{\lambda} M^{i j} q_{i}^{a} J_{a b} q_{j}^{b}$. The scale $\lambda$ is needed to match the dimensions of the fields and relates the scales $\Lambda_{N=1}$ and $\widetilde{\Lambda}_{N=1}$ of the electric and magnetic theories by $\Lambda_{N=1}^{3\left(N_{c}+1\right)-N_{f}} \widetilde{\Lambda}_{N=1}^{3\left(\widetilde{N}_{c}+1\right)-N_{f}}=(-1)^{N_{f}-N_{c}-1} \lambda^{N_{f}}$. In order to study the low energy behavior of our model, we may as well consider the magnetic theory with the tree level superpotential

$$
W_{\text {tree }}=\frac{1}{\lambda} M q q-\frac{1}{2 \mu} \operatorname{Tr}\left(M^{2}\right) .
$$

If the rank of $M$ is maximal, $\operatorname{rank} M=2 N_{f}$, all of the dual quarks are massive and the low energy physics is that of $S p\left(\widetilde{N}_{c}\right)$ Yang-Mills theory with the dynamical scale $\Lambda_{L}$ given by $\Lambda_{L}^{3\left(\widetilde{N}_{c}+1\right)}=\operatorname{Pf}(M / \lambda) \widetilde{\Lambda}_{N=1}^{3\left(\widetilde{N}_{c}+1\right)-N_{f}}$ and an effective superpotential given by

$$
W_{\text {eff }}=\left(N_{c}+1-N_{f}\right)\left(\frac{\operatorname{Pf} M}{\Lambda_{N=1}^{3\left(N_{c}+1\right)-N_{f}}}\right)^{1 /\left(N_{f}-N_{c}-1\right)}-\frac{1}{2 \mu} \operatorname{Tr}\left(M^{2}\right) .
$$


Proceeding as in [18], we find that the extremum of $W_{\text {eff }}$ with $\frac{1}{2} \operatorname{rank} M=N_{f}$ is given by

- $N_{f}$ odd

$$
M=\operatorname{diag}(\overbrace{m, \ldots, m, m}^{N_{f}}) \otimes \epsilon,
$$

- $N_{f}$ even

$$
M=\left\{\begin{array}{l}
\operatorname{diag}(m, \ldots, m, m) \otimes \epsilon, \\
\operatorname{diag}(m, \ldots, m,-m) \otimes \epsilon,
\end{array}\right.
$$

where

$$
m=2^{-\frac{N_{c}+1-N_{f}}{2 N_{c}+2-N_{f}}} \mu \Lambda_{N=2},
$$

or its rotation by the flavor and the R-symmetry group $S O\left(2 N_{f}\right) \times \mathbf{Z}_{2 N_{c}+2-N_{f}}$. For $N_{f}$ odd, there are $2 N_{c}+2-N_{f}$ distinct flavor orbits which are related by the R-symmetry. For $N_{f}$ even there are $N_{c}+1-N_{f} / 2$ flavor orbits related by the R-symmetry from the first type of solutions of (3.12) and the same number of orbits related by $\mathbf{Z}_{2 N_{c}+2-N_{f}}$ from the second type, and in total there are $2 N_{f}+2-N_{f}$ distinct flavor orbits. Each of the flavor orbits is the homogeneous space $S O\left(2 N_{f}, \mathbf{C}\right) / H_{\mathbf{C}}$ where $H_{\mathbf{C}}$ is the complexification of the unbroken subgroup of $S O\left(2 N_{f}\right)$ at the diagonal solution (3.11), (3.12). For (3.11) and the first type of (3.12), the unbroken subgroup is $H=U\left(N_{f}\right)$, whereas for the second type of (3.12) it is some other subgroup of the same dimension as $U\left(N_{f}\right)$. In any case, the homogeneous space has complex dimension $N_{f}\left(N_{f}-1\right)$.

For small values of $\mu$, this result must be consistent with the analysis based on the $N=2$ description. From the $\mathbf{Z}_{2 N_{c}+2-N_{f}}$ symmetry breaking pattern, these are to be identified with the $\mathbf{A}$ branch in the $N=2$ analysis. Recall that the $\mathbf{A}$ branch emanates from the locus where $r=\left[\left(N_{f}-1\right) / 2\right]$ of $\phi_{a}$ 's are vanishing. At first sight this suggests that the $\mathbf{A}$ branch is the $r=\left[\left(N_{f}-1\right) / 2\right]$ Higgs branch. For odd $N_{f}$ it is consistent because the dimension of the $r=\left(N_{f}-1\right) / 2$ Higgs branch has complex dimension $4 r N_{f}-2\left(2 r^{2}+r\right)=$ $N_{f}\left(N_{f}-1\right)$. However, there is a puzzle for even $N_{f}$ : The complex dimension of the $r=\left[\left(N_{f}-1\right) / 2\right]=\left(N_{f}-2\right) / 2$ Higgs branch is $4 r N_{f}-2\left(2 r^{2}+r\right)=N_{f}\left(N_{f}-1\right)-2$ which is smaller by 2 than the above result. We will solve this puzzle in the brane picture.

These are the only supersymmetric vacua for $N_{f} \leq N_{c}+1$. For $N_{f} \geq N_{c}+2$, there is another branch with $\frac{1}{2} \operatorname{rank} M<N_{f}$.

For $N_{f}=N_{c}+2$, we can consider (3.8) to be the exact superpotential which is valid for all values of the ranks of $M$. There is a unique solution of lowest rank of the extremum equation. It is

$$
M=0 .
$$


This new solution corresponds to the $\mathbf{B}$ branch of the $N=2$ analysis since the $\mathbf{B}$ branch is expected to be the $r=N_{f}-N_{c}-2=0$ Higgs branch which has dimension zero.

For $N_{f}>N_{c}+2$, we cannot consider (3.10) as the exact superpotential for nonmaximal $\operatorname{rank} M$. The classical flat direction, or the extremum locus of (3.9), is described as

$$
\operatorname{rank} M \leq 2 \widetilde{N}_{c}, \quad M^{2}=0,
$$

and $q \cdot q=(\lambda / \mu) M$. In this direction, at least $2 N_{f}^{\prime}=2 N_{f}-2 \widetilde{N}_{c}$ of the dual quarks are massless. Thus, the low energy theory is the $S p\left(\widetilde{N}_{c}\right)$ gauge theory with massless quarks $q_{\text {low }}^{i}, i=1, \ldots, 2 N_{f}^{\prime}$ (the flavor is larger for $\left.\operatorname{rank} M<2 \widetilde{N}_{c}\right)$. Note that $N_{f}^{\prime}-\left(\widetilde{N}_{c}+2\right)=$ $2\left(N_{c}+1\right)-N_{f}$ is positive because our starting point was an asymptotically free $N=2$ theory. Thus, the origin $q_{\text {low }}^{i}=0$ of the moduli space of the low energy theory is not lifted. This shows that the quantum moduli space contains the classical flat direction (3.15) as one of its branches. ${ }^{3}$ One can show as in [68] that there are no other branches. We note that the constraint (3.15) is the same as the one defining the Higgs branch of $N=2 \operatorname{Sp}\left(\widetilde{N}_{c}\right)$ gauge theory with $N_{f}$ flavors [55]. Thus, this branch is identified as the $\mathbf{B}$ branch of the $N=2$ analysis which is expected to be the $r=\widetilde{N}_{c}$ Higgs branch because $r=N_{f}-N_{c}-2=\widetilde{N}_{c}$ of the $\phi_{a}$ are vanishing at the root.

The $\mu \rightarrow \infty$ Limit

As $\mu \rightarrow \infty$ keeping $\Lambda_{N=1}$ finite, because

$$
\left(\mu \Lambda_{N=2}\right)^{2 N_{c}+2-N_{f}}=\mu^{N_{c}+1-N_{f}} \Lambda_{N=1}^{3 N_{c}+3-N_{f}},
$$

the branch with maximal $\operatorname{rank} \frac{1}{2} \operatorname{rank} M=N_{f}$ runs away to infinity for $N_{f}<N_{c}+1$, whereas for $N_{f} \geq N_{c}+1$ it remains finite and constitutes a submanifold of dimension $N_{f}\left(N_{f}-1\right)$ of the moduli space of SQCD.

The lower rank branch which is present for $N_{f} \geq N_{c}+2$ remains finite in the $\mu \rightarrow \infty$ limit and constitutes a submanifold $M^{2}=0, \frac{1}{2} \operatorname{rank} M \leq \widetilde{N}_{c}$ of dimension $2\left(2 \widetilde{N}_{c} N_{f}-\right.$ $\left.\left(2 \widetilde{N}_{c}^{2}+\widetilde{N}_{c}\right)\right)$ of the moduli space $\frac{1}{2} \operatorname{rank} M \leq N_{c}$ of SQCD.

\footnotetext{
${ }^{3}$ This is in contrast with the case of $S U\left(N_{c}\right)$ gauge theory with $N_{f}>N_{c}+1$ flavors and a heavy adjoint in which the analogous branch is described at low energy by the $S U\left(\widetilde{N}_{c}\right)$ gauge theory with $\widetilde{N}_{c}$ massless quarks. The quantum modification of the moduli space of this low energy theory [51] induces a modification of this branch $[68,18]$.
} 


\section{2 $M$ Theory Realization of Orientifold Four-Plane}

In the next subsection, we will construct a $M$ theory fivebrane configuration whose worldvolume dynamics describes at long distances the $S p\left(N_{c}\right)$ gauge theory with $N=2$ or $N=1$ supersymmetry. In the weakly coupled Type IIA regime, the configuration involves an orientifold four-plane (O4-plane). Here, we briefly explain the $M$ theory realization of an O4-plane in Type IIA string theory.

In Type IIA string theory, there are two types of O4-plane which are classified by the $\mathrm{RR}$ charges [57]. One type carries D4-brane charge -1 if it is counted before taking the $\mathbf{Z}_{2}$ quotient (and therefore, $-1 / 2$ after the quotient), while the other carries D4-brane charge +1 . When there are $2 n$ D4-branes close to the $\mathbf{Z}_{2}$ fixed plane of the former type there is a $S O(2 n)$ gauge symmetry in the common directions of the branes (which is generically broken to $U(1)^{n}$ spontaneously), while the gauge symmetry is $S p(n)$ for the latter type. For this reason, we shall call the former SO-type and the latter Sp-type. In other words, an open string ending on D4-branes at an $S O$-type O4-plane carries $S O(2 n)$ Chan-Paton factors while an open string ending on D4-branes at an $S p$-type O4-plane carries $S p(n)$ Chan-Paton factors.

An orientifold of Type IIA on $\mathbf{R}^{5} \times \mathbf{R}^{5} / \mathbf{Z}_{2}$ is obtained from $M$ theory on the orbifold $\mathbf{R}^{5} \times \mathbf{R}^{5} / \mathbf{Z}_{2} \times S^{1}$ by taking the limit where the radius of $S^{1}$ is small. $M$ theory on $\mathbf{R}^{6} \times T^{5} / \mathbf{Z}_{2}$ was studied in $[58,59]$ and it was concluded from the local cancellation of gravitational anomaly that each of the $\mathbf{Z}_{2}$ fixed plane must carry fivebrane charge -1 (in the unit before quotient). Since a fivebrane wrapped on $S^{1}$ becomes the D4 brane in the Type IIA limit, this shows that the O4-plane which is identified as the $\mathbf{Z}_{2}$ fixed plane compactified on $S^{1}$ carries D4-brane charge -1. Namely, the O4-plane obtained in this way is of $S O$-type.

Then, what is the $M$ theory interpretation of the $S p$-type O4-plane? Here we propose that it can be obtained by putting two fivebranes at the $\mathbf{Z}_{2}$ fixed point set $\mathbf{R}^{5} \times S^{1}$, and then taking the $\mathbf{Z}_{2}$ quotient such that the degrees of freedom corresponding to the motion of the fivebranes away from the $\mathbf{Z}_{2}$ fixed points are frozen. The D4-brane charge of the $\mathbf{Z}_{2}$ fixed plane in the Type IIA limit is now $-1+2=+1$, which agrees with the $S p$-type O4-plane. For further test of the idea, let us put $2 n$ additional D4-branes parallel and close to this $\mathbf{Z}_{2}$ fixed plane. Then, the light degrees of freedom on the worldvolume in the common $4+1$ dimensions are created by open strings ending on the $2 n+2 \mathrm{D} 4$-branes where +2 are the two D4-branes corresponding to the two fivebranes stuck at the $\mathbf{Z}_{2}$ fixed

point. Since the motion of the two D4-branes are frozen, degrees of freedom coming from the open strings with both ends at these two D4-branes are killed. Then, it is easy to 
see that the remaining light degrees of freedom have the same mass spectrum as those associated with the $N=2 S p(n)$ gauge theory in $4+1$ dimensions. Note however that we do not see directly how the above proposed definition of $S p$-type O4-plane indeed yields $S p$ gauge group.

In general, we must assume the existence of such a $\mathbf{Z}_{2}$ quotient that freezes the motion of the two fivebranes. However, in what follows we consider a configuration with NS 5branes transversal to the O4-plane in the Type IIA regime. The presence of such NS 5-branes naturally freezes the two D4 branes within the standard $\mathbf{R}^{5} / \mathbf{Z}_{2}$ orbifold of $M$ theory (which, in the absence of the fivebranes, would yield the $S O$-type O4-plane in the Type IIA limit). This follows from the basic hypothesis that a $t$-configuration is not supersymmetric, as we will now see.

\subsection{The Fivebrane Configuration}

\section{Type IIA Configuration}

We can realize the $S p\left(N_{c}\right)$ gauge theories as considered in the first subsection by configurations in a Type IIA orientifold on $\mathbf{R}^{5} \times \mathbf{R}^{5} / \mathbf{Z}_{2}$ which involve (before the $\mathbf{Z}_{2}$ quotient) two NS 5-branes, $2 N_{c}$ D4-branes parallel to the O4-plane, and $2 N_{f}$ D6-branes. We denote the time and space coordinates by $x^{0}$ and $x^{1}, \ldots, x^{9}$, where the $\mathbf{Z}_{2}$ acts as the sign change of $x^{4,5,7,8,9}$ so that the O4-plane spans the 01236 directions. The NS 5-branes are separated in the $x^{6}$ direction, the D4-branes are stretched between them, and the D6branes, spanning the 0123789 directions, are located between them. In the configuration corresponding to the $N=2$ theory, the two NS 5-branes are parallel and span the 012345 directions. Giving a mass to the adjoint corresponds to rotating one of them in the 45-89 direction [5], and sending the mass to infinity corresponds to taking the right angle limit, in which the rotated NS 5-brane spans the 012389 directions. See figure 1.

In any case, the O4-plane is separated into three pieces by the two NS 5-branes. In order to obtain $S p\left(N_{c}\right)$ gauge theory on the worldvolume of the D4 branes, we want the O4-plane to be of $S p$-type (D4-brane charge +1 ) in the part between the two NS 5-branes. Since the flavor group of the $N=2$ theory is $S O\left(2 N_{f}\right)$, the other two parts of the O4plane should be of $S O$-type (D4-brane charge -1 ) in the $N=2$ configuration [7]. This has been proven using a world-volume computation in [47]. The other two parts of the O4-plane must then also be of $S O$-type for all other configurations that can be obtained by a rotation of the $N=2$ configuration (and possibly by other continuous deformations). 


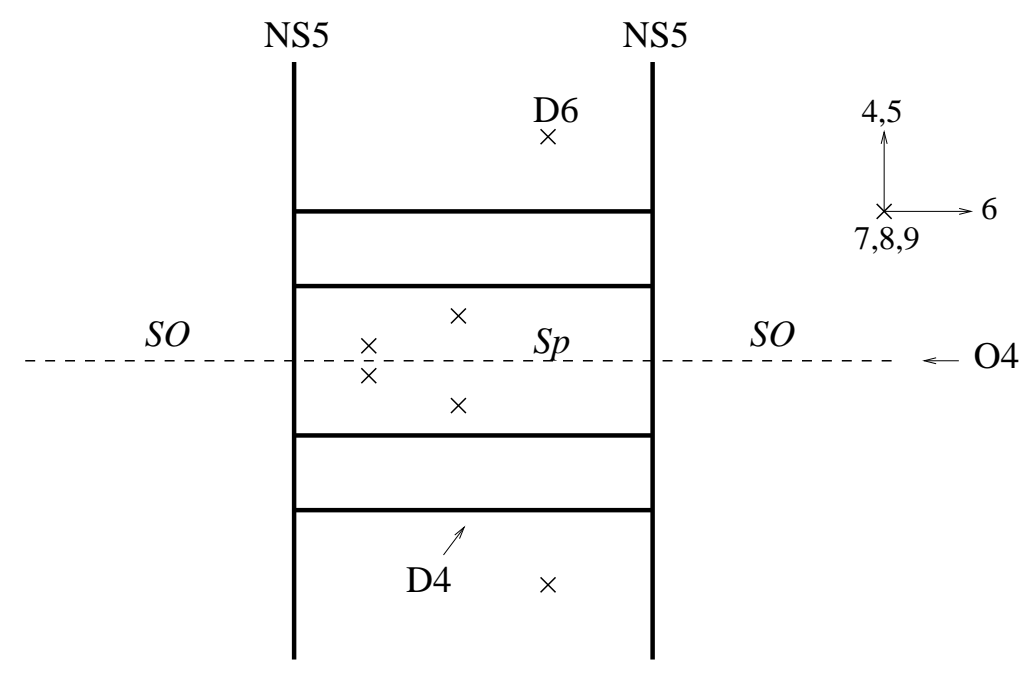

Figure 1: Type IIA Configuration

\section{$M$ Theory Realization of O4-NS5 System}

We first give an $M$ theory interpretation of the simplest configuration of two NS 5branes intersecting with the O4-plane of the type described right above. We consider the case of parallel NS5-branes spanning the 012345 directions. It is described by a configuration of a single fivebrane in $M$ theory on the orbifold $\mathbf{R}^{5} \times \mathbf{R}^{5} / \mathbf{Z}_{2} \times S^{1}$ and it is located at the origin in the 789 directions. We denote by $x^{10}$ the coordinate of the circle $S^{1}, x^{10} \equiv x^{10}+2 \pi$. Let us introduce the complex coordinates

$$
\begin{aligned}
& t=\exp \left(-\frac{x^{6}}{R}-i x^{10}\right), \\
& v=\left(x^{4}+i x^{5}\right) \times \text { const },
\end{aligned}
$$

where $R$ is the radius of $S^{1}$. The $\mathbf{Z}_{2}$ acts on these coordinates as $t \rightarrow t, v \rightarrow-v$.

There are two regions of the fivebrane worldvolume with large values of $v$ corresponding to the two NS 5-branes. Since the D4-brane charge jumps by +2 when crossing the left NS 5 -brane from left to right, the corresponding region of the fivebrane worldvolume behaves as $t \sim v^{2}$ at large $v$. Similarly, the charge jumps by -2 for the right NS5-brane and therefore the corresponding region behaves as $t \sim v^{-2}$ at large $v$. A general fivebrane invariant under the $\mathbf{Z}_{2}$ action $v \rightarrow-v$ which satisfies these boundary conditions is given by

$$
t^{2}-\left(v^{2}+c\right) t+\zeta=0,
$$

where $c$ and $\zeta$ are parameters with $\zeta \neq 0$. The coefficient of the $v^{2}$ term can be taken to 
be 1 by a rescaling of $t$ which is a shift of the origin of the $x^{6,10}$ directions. At first sight, there are two light degrees of freedom corresponding to the two moduli. Obviously, $\zeta$ is related to the distance between the two NS 5-branes and therefore can be considered as frozen (the kinetic energy for the variation of $\zeta$ diverges because of the infinite volume of the fivebrane). However, we still have one parameter $c$ which does not appear in the Type IIA configuration we want. Without the $\mathbf{Z}_{2}$ quotient this modulus would have a finite kinetic energy, as it is equivalent with the modulus of the Coulomb branch of $N=2$ super-Yang-Mills theory with gauge group $S U(2)$.

Now, this is the time where we can use the basic hypothesis to eliminate this degree of freedom. For generic values of $c$, the fivebrane (3.19) and the O4-plane $v=0$ intersect transversely at two points, each of which is a $t$-configuration. In the case

$$
c=2 \sqrt{\zeta}
$$

(3.19) looks $(t-\sqrt{\zeta})^{2} \sim v^{2}$ and we see that the two intersection points collide. If we accept the hypothesis that a $t$-configuration is not supersymmetric and two such intersection points attract each other, then the configuration is stable only in the case $c=2 \sqrt{\zeta}$. In this way, we can eliminate the degrees of freedom corresponding to varying $c$. $\ddagger$ From now on we will always assume that the intersection points attract each other for an $S O-S P-S O$-type O4-plane.

\subsection{1 $\quad N=2$ Configurations}

\section{Coulomb Branch}

We next review the $M$ theory fivebrane configuration describing the Coulomb branch of $N=2 S p\left(N_{c}\right)$ SQCD with $N_{f}$ massless flavors following [15]. In the Type IIA picture where all the $2 N_{f}$ D6-branes are sent to $x^{6}=+\infty$, there are $2 N_{f}$ semi-infinite D4branes ending on the right NS5-brane from the right. The $\mathbf{Z}_{2}$ invariant curve satisfying a suitable boundary condition is of the form $t^{2}-\left(v^{2 N_{c}+2}+u_{1} v^{2 N_{c}}+\cdots\right) t+\zeta v^{2 N_{f}}=0$. The requirement of absence of a $t$-configuration fixes one of the coefficients $u_{i}$ of the polynomial $v^{2 N_{c}+2}+u_{1} v^{2 N_{c}}+\cdots$, and it is easy to see that the curve for $N_{f} \neq 0$ is of the form

$$
t^{2}-v^{2} B_{N_{c}}\left(v^{2}\right) t+\Lambda_{N=2}^{4 N_{c}+4-2 N_{f}} v^{2 N_{f}}=0,
$$

\footnotetext{
${ }^{\ddagger}$ There are actually two choices of the sign of $\sqrt{\zeta}$. They are related by a coordinate change and therefore are equivalent. We will see some related phenomena in the following as we shall note in footnotes with the same symbol $\ddagger$.
} 
where $B_{N_{c}}=v^{2 N_{c}}+\sum_{i=1}^{N_{c}} u_{i} v^{2 N_{c}-2 i}$. For $N_{f}=0$, in order to avoid the $t$-configuration, $v^{2} B_{N_{c}}\left(v^{2}\right)$ must be replaced by $v^{2} B_{N_{c}}\left(v^{2}\right)+2 \Lambda_{N=2}^{2 N_{c}+2}$. ${ }^{\ddagger}$ If we identify $\Lambda_{N=2}$ and the $u_{i}$ 's with the dynamical scale and Casimirs of $\Phi$, this is the same as the Seiberg-Witten curve (3.2) describing the Coulomb branch of the $N=2$ gauge theory.

\section{Higgs Branch}

We next present a description of the $N=2$ Higgs branch in the brane picture. Because of our insufficient understanding of the strong coupling dynamics of Type IIA string theory in the presence of an orientifold four-plane and D6-branes, the description will be far from complete. ${ }^{1}$ However, by imposing some rule which generalizes our basic hypothesis, we can give a fairly reasonable description in terms of the fivebranes in $M$ theory on a certain orbifold. In particular, we can find a location of the $r=\left[N_{f} / 2\right]$ Higgs branch on the quantum Coulomb branch.

We consider taking the $\mathbf{Z}_{2}$ orbifold projection of $M$ theory on a Taub-NUT geometry which represents the $2 N_{f}$ D6-branes. In the case when all the D6-branes are at $v=0$, with a particular choice of complex structure the Taub-NUT space is described by

$$
x y=\Lambda_{N=2}^{4 N_{c}+4-2 N_{f}} v^{2 N_{f}}
$$

where $x$ and $y$ are complex coordinates. We can resolve the $A_{2 N_{f}-1}$ singularity at $x=$ $y=v=0$ by a complex surface covered by $2 N_{f}$ patches with coordinates $\left(y_{i}, x_{i}\right)(i=$ $\left.1, \ldots, 2 N_{f}\right)$ that are glued by $\left(y_{i-1}, x_{i-1}\right)=\left(y_{i}^{2} x_{i}, y_{i}^{-1}\right)$ and are mapped to $(y, x, v)$ as $y=\Lambda^{2 N_{c}+2-N_{f}} y_{i}^{i} x_{i}^{i-1}, x=\Lambda^{2 N_{c}+2-N_{f}} y_{i}^{2 N_{f}-i} x_{i}^{2 N_{f}+1-i}$, and $v=y_{i} x_{i}$. The singular point $x=y=v=0$ has been blown up to $2 N_{f}-1 \mathrm{CP}^{1}$ cycles $C_{i}$ defined by $y_{i}=x_{i+1}=0$ for $i=1, \ldots, 2 N_{f}-1$. The $\mathbf{Z}_{2}$ group acts on $(x, y, v)$ as $\rightarrow(x, y,-v)$ or on the coordinates of the $i$-th patch as $y_{i} \rightarrow(-1)^{i-1} y_{i}, x_{i} \rightarrow(-1)^{i} x_{i}$ (see figure 2 ). We see that the $\mathbf{Z}_{2}$ fixed plane has split into two infinite and $N_{f}-1 \mathrm{CP}^{1}$ cycles, namely, the infinite cigar at $x_{1}=0$, the cycles $C_{i}$ for even $i$, and another infinite cigar at $y_{2 N_{f}}=0$.

${ }^{\ddagger}$ Note that $2 \Lambda_{N=2}^{2 N_{c}+2}$ here can be replaced by $-2 \Lambda_{N=2}^{2 N_{c}+2}$. This means that the moduli space of holomorphic curves obeying the required condition splits into two disconnected components. However, the change of the sign of $\Lambda_{N=2}^{2 N_{c}+2}$ can be absorbed by a coordinate change, and the two connected components are actually equivalent. Thus, we can choose only one component as far as we consider small excitations around the supersymmetric vacua.

${ }^{1}$ This is in contrast with the case of an O6-plane and D6-branes where there is a complete geometric description in $M$ theory [60]. This leads to a nice description of the $N=2$ Coulomb and Higgs branches in terms of the fivebrane [61] (see also [31]). The results obtained for $N=2$ theories can actually be more straightforwardly obtained using an O6-plane. 


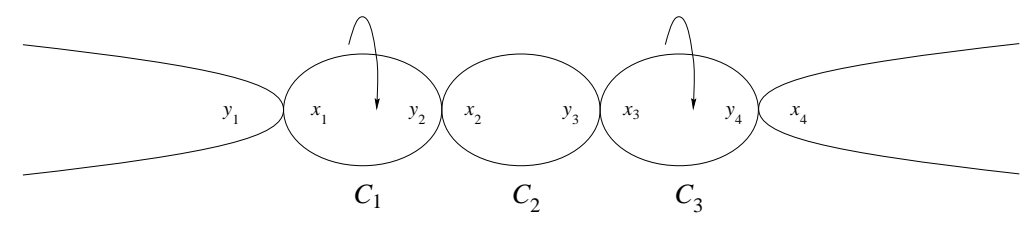

Figure 2: The $\mathbf{Z}_{2}$ action for $N_{f}=2$ case

The configuration of the fivebrane is given by

$$
x+y=v^{2} B_{N_{c}}\left(v^{2}\right)=v^{2} \prod_{a=1}^{N_{c}}\left(v^{2}-\phi_{a}^{2}\right) .
$$

This is obviously the same as (3.21) under the identification $y=t$.

Let us first look at a generic point of the Coulomb branch where $B_{N_{c}}\left(v^{2}\right)$ is not zero at $v=0$. As in [18], we see that the fivebrane wraps the $\mathrm{CP}^{1}$ cycles $C_{1}, C_{2}, \ldots, C_{2 N_{f}-2}, C_{2 N_{f}-1}$ with multiplicity $1,2, \ldots, 2,1$. Also, an infinite component intersects $C_{2}$ at one point and $C_{2 N_{f}-2}$ at one point (see figure 3 ). Recall that the $\mathbf{Z}_{2}$ acts on $\vec{w} \simeq\left(x^{7}, x^{8}, x^{9}\right)$ as $\vec{w} \rightarrow-\vec{w}$ at the same time. Only from the requirement of the $\mathbf{Z}_{2}$ invariance of the configuration, there is nothing to prevent the two components wrapped on $C_{i}, 2 \leq i \leq 2 N_{f}-2$, to be separated in the opposite direction of $\vec{w}$, which would correspond to some modulus of the worldvolume theory; from the transformation property under the $S U(2)$ R-symmetry it would be a hypermodulus. But this does not agree with the field theory knowledge; In $N=2 S p\left(N_{c}\right)$ SQCD, no Higgs branch emanates from a generic point of the Coulomb branch. Therefore, these degrees of freedom must be eliminated by some mechanism if the worldvolume theory is close to the $S p\left(N_{c}\right)$ gauge theory at all. We remark that the infi-

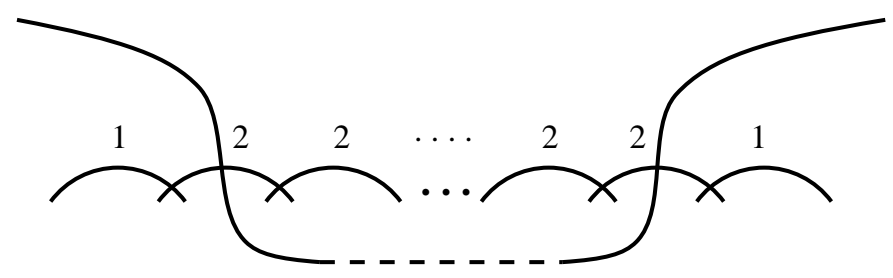

Figure 3: A Generic Point on the Coulomb Branch; The number above the $\mathbf{C P}^{1}$ components stands for the multiplicity.

nite component intersects $C_{2}$ and $C_{2 N_{f}-2}$ at one point each. An obvious way to eliminate these extra moduli is to generalize our basic hypothesis as follows. 
Suppose that the fivebrane intersects one or two different $\mathrm{CP}^{1}$ cycles of the $Z_{2}$ fixed plane at two distinct points. Then, the configuration is supersymmetric only if the two distinct intersection points are connected by one or more $\mathrm{CP}^{1}$ components of the fivebrane. Indeed, under the constraint that the intersection points at $C_{2}$ and $C_{2 N_{f}-2}$ be connected by a series of $\mathbf{C P}^{1}$ components, there is no $\mathbf{Z}_{2}$ invariant way to move the components wrapped on $C_{i}$ away from $\vec{w}=0$.

Let us next consider the case where $B_{N_{c}}\left(v^{2}\right)$ has a zero at $v=0$ of order $2 r$,

$$
B_{N_{c}}\left(v^{2}\right) \sim c v^{2 r}+\text { higher, }
$$

where $c$ is a non-zero constant. We first consider the range $r<\left[N_{f} / 2\right]$. Then, there are $\mathbf{C P}^{1}$ components wrapped on $C_{1}, \ldots, C_{2 N_{f}-1}$ with multiplicity $1,2,3, \ldots, 2 r+1,2 r+$ $2, \ldots, 2 r+2,2 r+1, \ldots, 3,2,1$ (there are $2 N_{f}-4 r-3 \mathbf{C P}^{1}$ cycles wrapped with multiplicity $2 r+2$ ), and also there is an infinite curve intersecting the first and last $\mathbf{C P}^{1}$ of multiplicity $2 r+2$. If we impose the generalized basic hypothesis, from each of the $\mathrm{CP}^{1}$ 's of multiplicity $2 r+2$ only $r$ pairs of components can be separated in the $\vec{w}$ direction. Thus, the number of separable pairs of $\mathrm{CP}^{1}$ components are

$$
\begin{aligned}
& 1+1+2+2+\cdots+r+r+\left(2 N_{f}-4 r-3\right) \times r+r+r+\cdots+1+1 \\
& =4 \frac{r(r+1)}{2}+\left(2 N_{f}-4 r-3\right) r=2 r N_{f}-\left(2 r^{2}+r\right),
\end{aligned}
$$

which agrees with the dimension of the $r$-th Higgs branch.

For the case $r=\left(N_{f}-1\right) / 2\left(N_{f}\right.$ odd $)$, the multiplicities of $\mathbf{C P}^{1}$ cycles are $1,2, \ldots, 2 r, 2 r+$ $1,2 r, \ldots, 2,1$ (there is no cycle with multiplicity $2 r+2$ ) and the infinite curve intersects the $\mathrm{CP}^{1}$ of multiplicity $2 r+1$. The number of separable pairs of $\mathbf{C P}^{1}$ components are $4 r(r-1) / 2+3 r=N_{f}\left(N_{f}-1\right) / 2$ which is the dimension of the $r=\left(N_{f}-1\right) / 2$ Higgs branch.

For higher $r$, the pattern of degeneration of the curve is the same as in the case of $r=\left(N_{f}-1\right) / 2$ (for $N_{f}$ odd) or $r=\left(N_{f}-2\right) / 2$ (for $N_{f}$ even).

So far, we have identified the $r=1,2, \ldots,\left[\left(N_{f}-1\right) / 2\right]$ Higgs branches. Then where is the $r=N_{f} / 2$ Higgs branch (in the case $N_{f}$ even)? Does it disappear in the quantum theory?

There is actually a subtlety in the case $r=\left(N_{f}-2\right) / 2\left(N_{f}\right.$ even $)$. In this case the number of $\mathrm{CP}^{1}$ 's with multiplicity $2 r+2$ is $2 N_{f}-4 r-3=1$. Thus, there is a possibility that the two intersection points of the infinite curve with the $\mathrm{CP}^{1}$ 's coincide. In that would happen, there would be no restriction from the generalized basic hypothesis concerning 
the separation of pairs of the $\mathrm{CP}^{1}$ components of the fivebrane. In order to see whether this is indeed what happens, let us look at the $N_{f}$-th patch with coordinate $\left(y_{N_{f}}, x_{N_{f}}\right)$. Equation (3.23) looks near $x=y=v=0$ as

$$
y_{N_{f}}^{N_{f}} x_{N_{f}}^{N_{f}-1}\left(x_{N_{f}}^{2}-\frac{c}{\Lambda_{N=2}^{2 N_{c}+2-N_{f}}} x_{N_{f}}+1\right)=0 .
$$

This shows in particular that the $\mathrm{CP}^{1}$ of multiplicity $2 r+2=N_{f}$ is the locus $y_{N_{f}}=0$. For generic values of $c$ the infinite component (described by $x_{N_{f}}^{2}-\frac{c}{\Lambda_{N=2}^{2 N_{c}+2-N_{f}}} x_{N_{f}}+1=0$ ) intersects the $\mathrm{CP}^{1}$ of multiplicity $2 r+2$ at two different points, and therefore at least one $\mathrm{CP}^{1}$ component of the fivebrane must be at $\vec{w}=0$ by the basic hypothesis. However, for

$$
c= \pm 2 \Lambda_{N=2}^{2 N_{c}+2-N_{f}}
$$

the two intersection points collide. Then the basic hypothesis imposes no restrictions, and we have one additional pair of $\mathrm{CP}^{1}$ components that can be separated, and the total number of movable pairs becomes

$$
2 r N_{f}-\left(2 r^{2}+r\right)+1=\frac{N_{f}\left(N_{f}-1\right)}{2} .
$$

which is the dimension of the $r^{\prime}=N_{f} / 2$ Higgs branch. We conclude that we have indeed found the $r=N_{f} / 2$ Higgs branch. Namely, the $r=N_{f} / 2$ Higgs branch, which emanates classically from the locus where $N_{f} / 2$ of the $\phi_{a}$ 's vanish, emanates in the quantum theory from the locus where only $\left(N_{f}-2\right) / 2$ of them vanish and the product of non-zero $\phi_{a}^{2}$ 's is $\pm 2 \Lambda^{2 N_{c}+2-N_{f}}$. This generalizes the quantum splitting of the Higgs branch root $u=0 \rightarrow$ $\pm 2 \Lambda^{2}$ in the $S U(2)$ gauge theory with $N_{f}=2$ flavors.

There remains a question of understanding the theory at the root of the $r=N_{f} / 2$ Higgs branch. As noted before, an $S p\left(N_{f} / 2\right)$ gauge theory with $N_{f}$ flavors is asymptotically free and becomes strongly coupled at the scale $\Lambda_{N=2}$. Is the theory at the root a new non-trivial fixed point? At least for the $N_{c}=1$ case, we know another description of the theory (as an $S U(2)$ gauge theory) and we know that the theory at the root is just an $N=2$ free QED with two electrons. This may suggest that in general the gauge group splits into $U(1)$ and $S p\left(N_{f} / 2-1\right)$ and the theory flows to a direct product of a free QED and the conformal field theory of $S p\left(N_{f} / 2-1\right)$ with $N_{f}$ flavors.

\subsection{2 $N=1$ Configurations}

Rotation by Finite Angles

Next, we construct the fivebrane configuration for the theory with an adjoint mass $\mu$. This corresponds to rotating, say, the left NS 5-brane in the 45-89 plane while keeping 
fixed the right NS 5-brane [5]. The left (right) NS 5-brane corresponds to the asymptotic region with large $v$ where the curve behaves as $t \sim v^{2 N_{c}+2}\left(t \sim v^{2 N_{f}-2 N_{c}-2}\right)$. In terms of the complex coordinate

$$
w=\left(x^{8}+i x^{9}\right) \times \text { const }
$$

the boundary condition at the left infinity $t \sim v^{2 N_{c}+2}$ reads

$$
w \sim \mu v
$$

while it is $w \sim 0$ at the right infinity $t \sim v^{2 N_{f}-2 N_{c}-2}$. We require that the configuration is invariant under the rotations $U(1)_{45}$ and $U(1)_{89}$ in the 45 and 89 planes which are identified with $U(1)$ R-symmetries under which $\Phi$ carries charge 2 and 0 respectively. Under the group $U(1)_{45} \times U(1)_{89}$, the coordinates and parameters are charged as $v$ : $(2,0), w:(0,2), \mu:(-2,2), t:\left(4 N_{c}+4,0\right), x:\left(4 N_{c}+4,0\right)$, and $\Lambda_{N=2}^{2 N_{c}+2-N_{f}}:\left(4 N_{c}+\right.$ $\left.4-2 N_{f}, 0\right)$. The combination $\frac{1}{2} U(1)_{45}+\frac{1}{2} U(1)_{89}$ makes $\mu$ invariant, and its $\mathbf{Z}_{2 N_{c}+2-N_{f}}$ subgroup makes $\Lambda_{N=2}^{2 N_{c}+2-N_{f}}$ invariant. The latter is interpreted as the non-anomalous $\mathbf{Z}_{2 N_{c}+2-N_{f}}$ R-symmetry of the field theory. As in [18], from the invariance under the $U(1)$ symmetries, we can conclude that the projection of the rotated curve is the same as the curve before rotation. Then, the $w$ values of the rotated curve can be considered as a function on a original, fixed curve.

Let us first consider the case where the curve has a single infinite component. When the curve is compactified at the two points with $v=\infty, w$ has a simple pole at one infinity by (3.30) and hence the compactified curve is necessarily (birational to) $\mathbf{C P}^{1}$, and the rotated curve is a cylinder which is globally parametrized by $w$. Proceeding as in [18], we can determine the allowed form of $t$ and $v$ as functions of $w$. The only difference from [18] is that here we require the curve to be invariant under the $\mathbf{Z}_{2}:(t, v, w) \rightarrow(t,-v,-w)$, and we find

$$
\begin{aligned}
v & =\mu^{-1} w^{-1}\left(w^{2}-M^{2}\right), \\
t & =\mu^{-2 N_{c}-2} w^{2 N_{c}+2-2 N_{f}}\left(w^{2}-M^{2}\right)^{N_{f}}
\end{aligned}
$$

By requiring that this projects to a curve in the $t$ - $v$ plane of the form (3.21), we find that for $N_{f}>0 M$ must satisfy the equation

$$
M^{4 N_{c}+4-2 N_{f}}=(-1)^{N_{f}}\left(\mu^{2} \Lambda_{N=2}^{2}\right)^{2 N_{c}+2-N_{f}} .
$$

There are $2 N_{c}+2-N_{f}$ solutions for $M^{2}$. The $\mathbf{Z}_{2 N_{c}+2-N_{f}}$ rotational symmetry is completely broken by any of such $M^{2}$ 's for $N_{f}$ odd while it is broken to $\mathbf{Z}_{2}$ for $N_{f}$ even, since its generator acts on $M^{2}$ as

$$
M^{2} \rightarrow \mathrm{e}^{\frac{4 \pi i}{2 N_{c}+2-N_{f}}} M^{2}
$$


For $N_{f}=0$, we find $M^{2 N_{c}+2}=\left(\mu^{2} \Lambda_{N=2}^{2}\right)^{N_{c}+1}$ and there are $N_{c}+1$ solutions for $M^{2}$ related by the $\mathbf{Z}_{2 N_{c}+2} \mathrm{R}$-symmetry.

These properties are the same as the structure of vacua with maximal rank solutions for the meson vev in the $N=1$ superpotential analysis, which are identified as the $\mathbf{A}$ branch of the $N=2$ analysis. Indeed, one can show that the function $B_{N_{c}}\left(v^{2}\right)$ defining the projected curve in the $t-v$ plane is of the form $B_{N_{c}}\left(v^{2}\right)=c v^{2 r}+\cdots$ (higher order terms), $c \neq 0$, where $r \geq\left(N_{f}-1\right) / 2$ for $N_{f}$ odd and $r=\left(N_{f}-2\right) / 2$ for $N_{f}$ even which is the property of the $\mathbf{A}$ branch. This can be seen as follows: Note that as $w \rightarrow \pm M$, both $v \rightarrow 0$ and $t \rightarrow 0$, because $t \sim v^{N_{f}}$ as can be seen from (3.31)-(3.32). On the other hand, equation (3.21) implies

$$
t \simeq \frac{c}{2} v^{2 r+2} \pm \sqrt{\left(\frac{c}{2} v^{2 r+2}\right)^{2}-\Lambda_{N=2}^{4 N_{c}+4-2 N_{f}} v^{2 N_{f}}} .
$$

The two statements are consistent only when $r \geq\left(N_{f}-2\right) / 2$. If $N_{f}$ is even, equations (3.31)-(3.32) show that $t$ is a single valued function of $v$ near $v \sim 0$ (for odd $N_{f}$, it is two-valued because the choice of $w=+M$ or $-M$ affects the sign of $t$ ). This is possible only if the two terms in the square root of (3.35) cancel, namely, when $r=\left(N_{f}-2\right) / 2$ and $c= \pm 2 \Lambda_{N=2}^{2 N_{c}+2-N_{f}}$.

Recall now that there was a puzzle in the field theory analysis for even $N_{f}$ concerning the dimension of the moduli space: The $\mathbf{A}$ branch emanates from the locus of the Coulomb branch where $r=\left(N_{f}-2\right) / 2$ of the $\phi_{a}$ vanish, and from this we expected that this branch is the $r=\left(N_{f}-2\right) / 2$ Higgs branch which has complex dimension $N_{f}\left(N_{f}-1\right)-2$. However, $N=1$ analysis shows that the dimension is $N_{f}\left(N_{f}-1\right)$. The solution of the puzzle is that the A branch is actually the $r=N_{f} / 2$ Higgs branch which, in the $N=2$ theory, emanates from the locus where $r=\left(N_{f}-2\right) / 2$ of the $\phi_{a}$ are vanishing and the product of the non-zero $\phi_{a}^{2}$ is $\pm 2 \Lambda^{2 N_{c}+2-N_{f}}$, as we have seen.

To summarize, the branch of vacua that remain after turning on a mass for the adjoint that we considered so far is identified with the branch in the $N=1$ superpotential analysis where the meson has maximal rank, and with the $\mathbf{A}$ branch of the $N=2$ analysis. In the $N=2$ limit, the $\mathbf{A}$ branch is the $r=\left[N_{f} / 2\right]$ Higgs branch.

Next, we consider the case where the curve is factorized so that the two infinities are separated. When such a factorization occurs, we just have to rotate the component including the left infinity by $w=\mu v$. It is easy to see that the factorization of $(3.21)$ is unique and is given by

$$
\left(t-v^{2 N_{c}+2}\right)\left(t-\Lambda_{N=2}^{4 N_{c}+4-2 N_{f}} v^{2 N_{f}-2 N_{c}-2}\right)=0 .
$$


By expanding this in $t$, we see that $v^{2} B_{N_{c}}\left(v^{2}\right)=v^{2 N_{c}+2}+\Lambda_{N=2}^{4 N_{c}+4-2 N_{f}} v^{2 N_{f}-2 N_{c}-2}$. In particular, this factorization is possible only for $N_{f} \geq N_{c}+2$. Also, this shows that the curve belongs to the $r=N_{c}-N_{f}-2$ Higgs branch root. Therefore, we can identify this as the $\mathbf{B}$ branch of the $N=2$ analysis which is identified with the branch in the $N=1$ analysis with non-maximal rank meson vev's.

The Limit $\mu \rightarrow \infty$

We consider taking the $\mu \rightarrow \infty$ limit. As in the case of $S U\left(N_{c}\right)$ gauge group [18], we need to rescale the coordinate as $\widetilde{y}(=\widetilde{t})=\mu^{2 N_{c}+2} t$ in order to not let the configuration run away. The complex structure of the space-time (before the $\mathbf{Z}_{2}$ quotient) is described by $\widetilde{y} x=\Lambda_{N=1}^{6 N_{c}+6-2 N_{f}} v^{2 N_{f}}$, where $\Lambda_{N=1}^{3 N_{c}+3-N_{f}}=\mu^{N_{c}+1} \Lambda_{N=2}^{2 N_{c}+2-N_{f}} . \Lambda_{N=1}^{3 N_{c}+3-N_{f}}$ is invariant under the $\mathbf{Z}_{2 N_{c}+2-2 N_{f}}\left(\mathbf{Z}_{2 N_{c}+2}\right)$ subgroup of $U(1)_{45}\left(U(1)_{89}\right)$.

For $N_{f}=0$, the limit of one of the $N_{c}+1$ curves is given by

$$
\begin{aligned}
& \tilde{t}=w^{2 N_{c}+2}, \\
& v w=-\Lambda_{N=1}^{3},
\end{aligned}
$$

and the limits of the other $N_{c}$ curves are given by the action of the $\mathbf{Z}_{2 N_{c}+2}$ symmetry. This in particular exhibits the chiral symmetry breaking $\mathbf{Z}_{2 N_{c}+2} \rightarrow \mathbf{Z}_{2}$.

For $0<N_{f}<N_{c}+1$, the curve becomes infinitely elongated because $M^{2} \rightarrow \infty$ in the limit. This is consistent with the absence of supersymmetric vacua for this number of flavors in the field theory.

For $N_{f} \geq N_{c}+1$, the limit exists. It contains two infinite components and several $\mathrm{CP}^{1}$ components. The infinite components $-C_{L}$ and $C_{R}-$ are given by

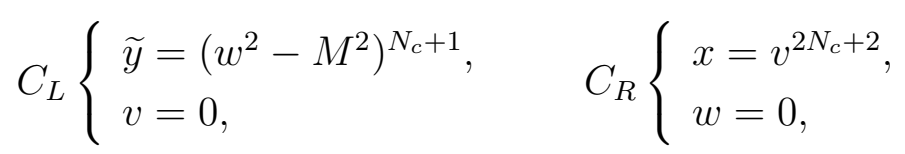

where $\left(M^{2}\right)^{N_{c}+1}=\left(-\Lambda_{N=1}^{4}\right)^{N_{c}+1}$ for $N_{f}=N_{c}+1$ while $M^{2}=0$ for $N_{f}>N_{c}+1$. The $\mathrm{CP}^{1}$ components are of multiplicity $2 N_{c}+2, \ldots, 2 N_{c}+2,2 N_{c}+1,2 N_{c}, \ldots, 3,2,1$ from left to right (the number of $2 N_{c}+2$ 's is $2 N_{f}-2 N_{c}-2$ ). For $N_{f} \geq N_{c}+2$, the component $C_{L}$ intersects the left-most $\mathbf{C P}^{1}$ at $w=0$ and the component $C_{R}$ intersects the last $\mathbf{C P}^{1}$ transversely with multiplicity $2 N_{c}+2$. For $N_{f}=N_{c}+1$, both do not intersect any of the $\mathrm{CP}^{1}$ 's at $w=0$. 
Configuration for $N=1 S Q C D$

After the limit $\mu \rightarrow \infty$, the curve acquires new deformation directions for $N_{f} \geq N_{c}+1$. The component $C_{R}$ is still rigid but $C_{L}$ can be deformed as $\widetilde{y}=\left(w^{2}-M_{1}^{2}\right) \cdots\left(w^{2}-M_{N_{c}+1}^{2}\right)$, $v=0$. However, there is a constraint for the allowed values of $M_{i}^{2}$ which is required by the basic hypothesis.

Let us first consider the case of $N_{f}=N_{c}+1$. In this case, the component $C_{R}$ can be described as $\widetilde{y}=\Lambda_{N=1}^{4 N_{c}+4}, w=0$, and hence it intersects the $\mathbf{Z}_{2}$ fixed point set at one point $(x, \widetilde{y}, v, w)=\left(0, \Lambda_{N=1}^{4 N_{c}+4}, 0,0\right)$. This is a $t$-configuration and it is not supersymmetric by itself according to the basic hypothesis. The intersection point attracts the other infinite component $C_{L}$ so that $C_{L}$ intersects the $\mathbf{Z}_{2}$ fixed point set at the same point. This is equivalent to requiring

$$
M_{1}^{2} \cdots M_{N_{f}}^{2}=(-1)^{N_{c}+1} \Lambda_{N=1}^{4 N_{c}+4} .
$$

This corresponds to the quantum modified constraint Pf $M=\Lambda_{N=1}^{2 N_{c}+2}$ on the meson matrix.

In the case $N_{f} \geq N_{c}+2$, the component $C_{R}$ intersects transversely with the $2 N_{c}+2$ nd $\mathrm{CP}^{1}$ from the right. From the generalized basic hypothesis, there must be a chain of $\mathrm{CP}^{1}$ components that connects $C_{R}$ with $C_{L}$. Since $C_{R}$ is at $w=0$, all such $\mathbf{C P}^{1}$ components should also be at $w=0$ and in particular $C_{L}$ must intersect the left-most $\mathbf{C P}^{1}$ at $w=$ 0 . This means that some of the $M_{i}^{2}$ 's must be zero. This corresponds to the classical constraint $\operatorname{rank} M \leq 2 N_{c}$ which is also the full constraint in the quantum theory.

One can also see the agreement in the dimension of the moduli space. For $N_{f} \geq N_{c}+2$ there is a constraint for the motion of $\mathrm{CP}^{1}$ 's due to the generalized basic hypothesis, while there is no constraint on the $\mathrm{CP}^{1}$ motion for $N_{f}=N_{c}+1$. In either case the dimension is the sum of $N_{c}$ from the variation of the $M_{i}$ and $2\left(1+1+\cdots+N_{c}+N_{c}+\left(2 N_{f}-2 N_{c}-2\right) \times N_{c}\right)$ from the $\mathrm{CP}^{1}$ motion, and is in total

$$
N_{c}+4 \frac{N_{c}\left(N_{c}+1\right)}{2}+2\left(2 N_{f}-2 N_{c}-2\right) \times N_{c}=4 N_{f} N_{c}-\left(2 N_{c}^{2}+N_{c}\right),
$$

which agrees with what we know from field theory. 


\section{Izawa-Yanagida-Intriligator-Thomas Model}

\subsection{Field Theory Analysis}

The Model

In $[49,50]$, a non-chiral model of dynamical supersymmetry breaking with stable non-supersymmetric vacua was given. Classically it is defined as an $N=1$ supersymmetric $S p\left(N_{c}\right)$ gauge theory with $2 N_{f}=2\left(N_{c}+1\right)$ fundamental chiral multiplets $Q_{a}^{i}$ $\left(a=1, \ldots, 2 N_{c}, i=1, \ldots, 2 N_{f}\right)$ and a gauge singlet chiral multiplet $S_{i j}$ which is an antisymmetric tensor with respect to the flavor indices $i, j$. The tree level superpotential is given by

$$
W_{\text {tree }}=\lambda S_{i j} Q^{i} Q^{j}
$$

where $Q^{i} Q^{j}=J^{a b} Q_{a}^{i} Q_{b}^{j}$ form the basis of gauge invariant chiral superfields, ${ }^{1}$ and $\lambda$ is the Yukawa coupling constant. The coupling (4.1) is chosen so that the $S U\left(2 N_{f}\right)$ flavor symmetry is preserved. In this paper, we will consider a deformation of this model by a linear term in $S$ in the superpotential

$$
W_{\text {tree }}=\lambda S_{i j} Q^{i} Q^{j}-m J^{i j} S_{i j}
$$

which breaks the flavor symmetry to $S p\left(N_{f}\right)$. We shall denote this theory also by the IYIT model $[49,50]$, although the deformation by the linear term was not considered there.

The exact effective superpotential of the theory is given by

$$
W_{e f f}=X\left(\operatorname{Pf} M-\Lambda^{2 N_{c}+2}\right)+\lambda S_{i j} M^{i j}-m J^{i j} S_{i j}
$$

where $M^{i j}=-M^{j i}$ is the meson matrix corresponding to $Q^{i} Q^{j}$ and $\Lambda$ is the dynamical scale of the $S p\left(N_{c}\right)$ gauge interaction. $X$ in the first term is a chiral superfield which plays the role of a Lagrange multiplier. The variation of $W_{\text {eff }}$ with respect to $X, S$ and $M$ yields

$$
\begin{aligned}
\operatorname{Pf} M & =\Lambda^{2 N_{c}+2}, \\
M & =\frac{m}{\lambda} J \\
S & \propto M^{-1} .
\end{aligned}
$$

\footnotetext{
${ }^{1} J^{a b}$ is the skew-symmetric matrix of size $2 N_{c} \times 2 N_{c}$ which is preserved by the group $S p\left(N_{c}\right)$. Likewise, we will use $J^{i j}$ to denote the $S p\left(N_{f}\right)$ invariant matrix of size $2 N_{f} \times 2 N_{f}$. In a symplectic basis, the matrix $J=\left(J^{i j}\right)$ is given by $J=\mathbf{1}_{N_{f}} \otimes \epsilon$ where $\mathbf{1}_{N_{f}}$ is the identity matrix of size $N_{f}$ and $\epsilon=\left(\begin{array}{cc}0 & -1 \\ 1 & 0\end{array}\right)$.
} 
The first equation is the quantum modified constraint which represents the $S p\left(N_{c}\right)$ gauge dynamics in the case of $N_{f}=N_{c}+1$ [52]. The first two conditions (4.4) and (4.5) are consistently satisfied only if $\operatorname{Pf}(m J / \lambda)=\Lambda^{2 N_{c}+2}$, namely

$$
\left(\frac{m}{\lambda}\right)^{N_{c}+1}=\Lambda^{2 N_{c}+2}
$$

Therefore, if the condition (4.7) is not satisfied, the supersymmetry is spontaneously broken $[49,50]$.

If (4.7) is satisfied, the supersymmetry is not broken. Indeed, the meson matrix has a fixed vev $M=m J / \lambda$ satisfying the quantum modified constraint (4.4), and there is a complex one-dimensional flat direction for the values of $S$ because the condition (4.6) implies $S_{i j} \propto J_{i j}$.

Existence Of Non-supersymmetric Stable Vacuum

Let us analyze the model with $(m / \lambda)^{N_{c}+1} \neq \Lambda^{2 N_{c}+2}$ where the supersymmetry is dynamically broken. For simplicity, we consider the original model of $[49,50]$ where $m=0$, but the same analysis applies also to the case $m \neq 0$.

For large values of $S$, the fundamental chiral multiplets are heavy and the theory at energies below $\lambda S$ is the pure $N=1$ super-Yang-Mills theory whose dynamical scale $\Lambda_{L}$ is given by $\Lambda_{L}^{3 N_{c}+3}=\operatorname{Pf}(2 \lambda S) \Lambda^{2 N_{c}+2}$. The superpotential of the low energy theory is given by

$$
W_{L}=\left(N_{c}+1\right) \Lambda_{L}^{3}=\left(N_{c}+1\right) \operatorname{Pf}(2 \lambda S)^{1 /\left(N_{c}+1\right)} \Lambda^{2} .
$$

Around the locus $S_{i j}=\sigma J_{i j}$, all the components of $S_{i j}$ other than $\delta \sigma J_{i j}$ are massive, and the effective superpotential with respect to $\sigma$ is given by

$$
W_{L}=2\left(N_{c}+1\right) \lambda \sigma \Lambda^{2}
$$

We see that the supersymmetry is indeed broken in this direction $\partial W_{L} / \partial \sigma \sim \lambda \Lambda^{2} \neq 0$.

The scalar potential is thus given by

$$
U=g^{\sigma \bar{\sigma}}\left|\frac{\partial W_{L}}{\partial \sigma}\right|^{2}=g^{\sigma \bar{\sigma}}\left|2\left(N_{c}+1\right) \lambda \Lambda^{2}\right|^{2},
$$

where $g^{\sigma \bar{\sigma}}$ is the inverse of the Kähler metric $g_{\sigma \bar{\sigma}}$ with respect to $\sigma$. At large values of $\sigma$, we can evaluate the metric $g_{\sigma \bar{\sigma}}$ by perturbation theory. The dominant correction is due 
to the one loop of the $S p\left(N_{c}\right)$ fundamental chiral multiplets, and the corrected metric is given by

$$
g_{\sigma \bar{\sigma}}^{1-\mathrm{loop}}=1-\frac{N_{c}}{4 \pi^{2}}|\lambda|^{2} \log \left|\frac{\lambda \sigma}{M_{\mathrm{UV}}}\right|,
$$

where $M_{\mathrm{UV}}$ is the ultra-violet cut off. As we increase $\sigma, g_{\sigma \bar{\sigma}}^{1-\text { loop }}$ decreases, and hence the scalar potential (4.10) grows. ${ }^{2}$ Since the scalar potential grows at large values of $|\sigma|$, there must be a minimum at some smaller values of $\sigma$. This ensures the existence of a stable vacuum. ${ }^{3}$ If there is no singularity in the Kähler metric, the vacuum has finite energy and breaks supersymmetry.

The sign - (minus) of the one-loop correction (4.11) is essential for the potential growth at large $\sigma$. This would not have been the case if, say, we had gauged the $S O\left(2 N_{f}\right)$ subgroup of the flavor group as we will do in the next section.

\section{Perturbation By Quadratic Term}

For later use, we also consider a deformation of the superpotential (4.2) by a quadratic term in $S$ :

$$
\Delta W=\frac{1}{2 \mu} S_{i j} S^{i j}
$$

where the flavor indices are raised and lowered by the Kronecker delta $\delta^{i j}$ which is an $S O\left(2 N_{f}\right)$ invariant. This breaks the flavor symmetry further down to $S p\left(N_{f}\right) \cap S O\left(2 N_{f}\right)=$ $U\left(N_{f}\right)$. The effective superpotential of the deformed theory is given by

$$
W_{e f f}=X\left(\operatorname{Pf} M-\Lambda^{2 N_{c}+2}\right)+\lambda S_{i j} M^{i j}-m J^{i j} S_{i j}+\frac{1}{2 \mu} S_{i j} S^{i j}
$$

\footnotetext{
${ }^{2}$ Strictly speaking, the one-loop correction (4.11) is reliable for large $\sigma$ such that $|\lambda \sigma|$ is almost close to the cut off $M_{\mathrm{UV}}$. However, a simple renormalization group argument [53] shows that this potential rise persists for much smaller values of $\sigma$.

${ }^{3}$ More rigorously, we must show that the potential grows in all possible directions in the $S_{i j}$ space (at large values). This seems also to be true. Consider for example the case where $\left(S_{i j}\right)=\operatorname{diag}\left(\sigma_{1}, \ldots, \sigma_{N_{f}}\right) \otimes$ $\epsilon$ in which first $r \sigma_{i}$ 's are much smaller than the last $N_{f}-r: \sigma_{1} \sim \sigma_{2} \sim \cdots \sim \sigma_{r} \sim \sigma_{\text {small }} \ll \sigma_{\text {large }} \sim$ $\sigma_{r+1} \sim \cdots \sim \sigma_{N_{f}}$. Then, the superpotential of the effective theory at energies below $\lambda \sigma_{\text {large }}$ is given by

$$
W_{L} \sim\left(\sigma_{\text {small }}\right)^{r /\left(N_{c}+1\right)} .
$$

It gives the run-away potential with respect to $\sigma_{\text {small }}$ since $\partial W_{L} / \partial \sigma_{\text {small }} \sim\left(\sigma_{\text {small }}\right)^{r /\left(N_{c}+1\right)-1}$ is a negative power of $\sigma_{\text {small }}$ and the potential cannot be made to grow by a perturbative correction. Thus, $\sigma_{\text {small }}$ runs away to larger values until it becomes comparable to $\sigma_{\text {large }}$. We thank H. Murayama for explaining us this argument.
} 
The extremum condition reads as

$$
\begin{aligned}
& \operatorname{Pf} M=\Lambda^{2 N_{c}+2} \\
& M^{i j}-\frac{m}{\lambda} J^{i j}=\frac{1}{\lambda \mu} S^{i j} \\
& \lambda S_{i j}+\frac{1}{2} X \operatorname{Pf} M\left(M^{-1}\right)_{i j}=0 .
\end{aligned}
$$

The last two equations yield ${ }^{4}$

$$
M-\frac{m}{\lambda} J \propto M^{-1} .
$$

This in particular implies that the anti-symmetric matrix $M$ commutes with $J$, which shows that it can be expressed as

$$
M=A \otimes \epsilon+B \otimes \mathbf{1}_{2}
$$

where $\mathbf{1}_{2}$ is the $2 \times 2$ identity matrix and $A$ and $B$ are $N_{f} \times N_{f}$ symmetric and antisymmetric matrices respectively, ${ }^{t} A=A,{ }^{t} B=-B$. In other words, $M$ can be mapped to an $N_{f} \times N_{f}$ matrix $\widehat{M}=A-i B$ which transforms in the (complexified) adjoint representation of the flavor group $U\left(N_{f}\right)$. The equations (4.14) and (4.17) then yield

$$
\begin{aligned}
& \operatorname{det} \widehat{M}=\Lambda^{2 N_{c}+2} \\
& \widehat{M}^{2}-\frac{m}{\lambda} \widehat{M} \propto \mathbf{1}_{N_{f}} .
\end{aligned}
$$

As in [18], we can show that $\widehat{M}$ is diagonalizable for generic values of $m,{ }^{5}$ and there are at most two kinds of eigenvalues. Proceeding as in [18], we see that there are solutions parametrized by $r=0,1, \ldots,\left[N_{f} / 2\right]$, where the solution of the type $r$ is such that $r$ of the eigenvalues of $\widehat{M}$ are $M_{+}$and $N_{f}-r$ of them are $M_{-}$where

$$
\left(M_{+}\right)^{r}\left(M_{-}\right)^{N_{f}-r}=\Lambda^{2 N_{c}+2}, \quad M_{+}+M_{-}=\frac{m}{\lambda} .
$$

A general solution can be obtained from the complexified flavor rotation of a diagonal solution, and hence the moduli space of type $r$ solutions is the homogeneous space $G L\left(N_{f}, \mathbf{C}\right) /\left(G L(r, \mathbf{C}) \times G L\left(N_{f}-r, \mathbf{C}\right)\right)$ which is of complex dimension $2 r\left(N_{f}-r\right)$.

The vev of $S_{i j}$ is determined by $M$ through (4.15), $S=\lambda \mu(M-m J / \lambda)$ :

$$
S=\operatorname{diag}(\overbrace{\sigma_{+}, \ldots, \sigma_{+}}^{r}, \overbrace{\sigma_{-}, \ldots, \sigma_{-}}^{N_{f}-r}) \otimes \epsilon
$$

\footnotetext{
${ }^{4}$ Here and in what follows, the obvious multiplication by a Kronecker delta is abbreviated. For example, the matrix symbol $M$ means $M^{i j}$ as well as $M_{j}^{i}$ (or $M_{i j}$ ) and what it means should be clear from the context.

${ }^{5}$ Non-diagonalizable solutions are possible only for $(m / 2 \lambda)^{N_{c}+1}=\Lambda^{2 N_{c}+2}$ where there are solutions with Jordan blocks of size two.
} 
where $\sigma_{ \pm}=\lambda \mu\left(M_{ \pm}-m / \lambda\right)=-\lambda \mu M_{\mp}$ and thus

$$
\left(\sigma_{+}\right)^{N_{f}-r}\left(\sigma_{-}\right)^{r}=\left(-\lambda \mu \Lambda^{2}\right)^{N_{c}+1}, \quad \sigma_{+}+\sigma_{-}=-\mu m .
$$

As we take the limit $\mu \rightarrow \infty$, either $\sigma_{+}$or $\sigma_{-}$diverges, and hence $S$ runs away to infinity unless $r=0$ and $\sigma_{-}=$finite. This is possible if and only if the parameter $m$ satisfies the constraint $(m / \lambda)^{N_{c}+1}=\Lambda^{2 N_{c}+2}$. This is consistent with the constraint (4.7) for the existence of supersymmetric vacuum for the theory without the quadratic term (4.12). The vacuum obtained in this way has the vevs $M=m J / \lambda, S=0$. Other vacua with general $S$ can be obtained by tuning $m$ to approach $\Lambda^{2}$ as $m=\lambda \Lambda^{2}-\sigma / \mu$. Then the $r=0$ solution converges in the limit $\mu \rightarrow \infty$ to $M=m J / \lambda, S=\sigma J$.

Let us consider the case $(m / \lambda)^{N_{c}+1} \neq \Lambda^{2\left(N_{c}+1\right)}$ where the supersymmetric vacua run away to infinity $S \rightarrow \infty$ as $\mu \rightarrow \infty$. We have seen in the $\mu=\infty$ case that there is a (presumably non-supersymmetric) vacuum at a small value of $S$. Is there a local minimum for finite $\mu$ which approaches this vacuum in the $\mu \rightarrow \infty$ limit? Let us again look at the region of large $S$ with $S_{i j}=\sigma J_{i j}$. The effective superpotential is given by

$$
W_{\text {eff }}=2\left(N_{c}+1\right)\left(\sigma\left(\lambda \Lambda^{2}-m\right)-\frac{\sigma^{2}}{2 \mu}\right) .
$$

By taking into account the one-loop correction to the Kähler metric of $S_{i j}$ for large values of $\sigma$ so that $|\lambda \sigma|$ is almost close to the ultra-violet cut off $M_{\mathrm{UV}}$, the effective scalar potential is given by

$$
U=4\left(N_{c}+1\right)^{2}\left|\left(\lambda \Lambda^{2}-m\right)-\frac{\sigma}{\mu}\right|^{2}\left(1-\frac{N_{c}}{4 \pi^{2}}|\lambda|^{2} \log \left|\frac{\lambda \sigma}{M_{\mathrm{UV}}}\right|\right)^{-1}
$$

In the direction of the $\sigma$-plane where $\sigma$ has the same phase as $\mu\left(\lambda \Lambda^{2}-m\right)$, the first factor has a negative slope in the region $\sigma<\mu\left(\lambda \Lambda^{2}-m\right)$ while the second factor has a positive slope around $|\lambda \sigma| \lesssim M_{\mathrm{UV}}$. Therefore, whether $U$ is rising or not is a consequence of the conflict of the two factors. It turns out that it is rising near $|\lambda \sigma| \lesssim M_{\mathrm{UV}}$ (and hence there is a local minimum at smaller values) when $\mu$ is large enough so that

$$
\lambda \mu\left(\lambda \Lambda^{2}-m\right)>\left(\frac{8 \pi^{2}}{N_{c}|\lambda|^{2}}+1\right) M_{\mathrm{UV}}
$$

For illustration, we present in figure 4 the graph of the scalar potential $U$ (4.25), up to an overall normalization, for the case $M_{\mathrm{UV}}=1, \lambda \mu\left(\lambda \Lambda^{2}-m\right)=10, N_{c}|\lambda|^{2} / 4 \pi^{2}=1 / 3$, where the horizontal axis parametrizes $s=\lambda \sigma$. Note that the function (4.25) makes sense as scalar potential only in the region below the cut-off $\lambda \sigma \leq M_{\mathrm{UV}}$, and hence only the region $s \leq 1$ is important although we continued it to larger values. 


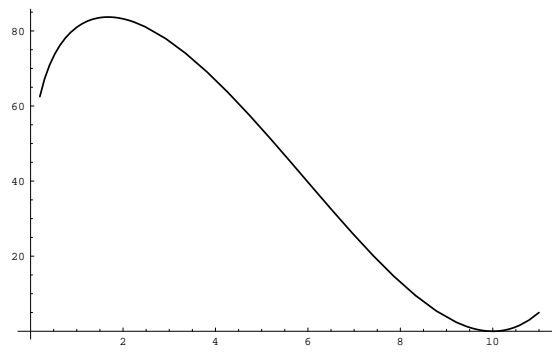

Figure 4: Graph of $(10-s)^{2} /\left(1-\frac{1}{3} \log s\right)$

\subsection{Brane Realization of the Model}

We construct a brane configuration corresponding to the IYIT model. We first construct the configuration for the model perturbed by the quadratic term $\Delta W=S^{2} / \mu$ which contains supersymmetric vacua, and then consider the limit $\mu \rightarrow \infty$.

\section{Configuration for Finite $\mu$}

We first note that the tree-level superpotential of the theory

$$
W_{\text {tree }}=\lambda S_{i j} Q^{i} Q^{j}-m J^{i j} S_{i j}+\frac{1}{2 \mu} S_{i j} S^{i j}
$$

is the same as the tree-level superpotential of the magnetic dual of the $S p\left(\widetilde{N}_{c}\right)$ gauge theory (which we shall call the electric dual) with $2 N_{f}$ quarks $q_{i}$ of bare mass $m$ with a quartic superpotential $(q q)^{2} / 2 \mu$. Although $\widetilde{N}_{c}=N_{f}-N_{c}-2=-1$, we can still make use of this fact as a guide to construct the brane configuration corresponding to the perturbed IYIT model $^{1}$. Namely, we first consider $N_{f}, N_{c}$ to be in the region where the electric-magnetic duality holds and then take the limit $\widetilde{N}_{c} \rightarrow-1$ after construction of the configuration. We then read off the boundary condition satisfied by the curves obtained in this way, and identify all the curves with the same boundary condition. As evidence we will show how to reproduce some of the results of section 4.1 from these brane configurations.

The meson field $q_{i} q_{j}$ of the electric dual is identified with $S_{i j}$. The tree-level superpotential $-m q q+(q q)^{2} / 2 \mu$ shows that this theory is obtained from the $N=2 S p\left(\widetilde{N}_{c}\right)$ SQCD with $N_{f}$ flavors by giving a bare mass $\mu$ to the adjoint chiral multiplet. Note that the dynamical scale $\widetilde{\Lambda}$ of the electric dual is given by $\Lambda^{3\left(N_{c}+1\right)-N_{f}} \widetilde{\Lambda}^{3\left(\widetilde{N}_{c}+1\right)-N_{f}}=(-1)^{\widetilde{N}_{c}+1} \lambda^{-N_{f}}$

\footnotetext{
${ }^{1}$ Recently, this procedure was justified in [68] by showing that the configuration constructed in this way correctly reproduces the IYIT model in the type IIA limit.
} 
and it is related to the dynamical scale $\widetilde{\Lambda}_{N=2}$ of the high energy $N=2$ theory by $\widetilde{\Lambda}^{3\left(\widetilde{N}_{c}+1\right)-N_{f}}=\mu^{\widetilde{N}_{c}+1} \widetilde{\Lambda}_{N=2}^{2\left(\widetilde{N}_{c}+1\right)-N_{f}}$.

The configuration for $\mu=0$ is given by

$$
t^{2}-\left(v^{2} B_{\widetilde{N}_{c}}\left(v^{2}\right)+2 \widetilde{\Lambda}_{N=2}^{2 \widetilde{N}_{c}+2-N_{f}} m^{N_{f}}\right) t+\widetilde{\Lambda}_{N=2}^{4 \widetilde{N}_{c}+4-2 N_{f}}\left(v^{2}+m^{2}\right)^{N_{f}}=0 .
$$

In the standard way, we can find the configuration for finite $\mu$. The result is

$$
\begin{aligned}
v w & =\mu^{-1}\left(w^{2}+w_{+} w_{-}\right) \\
t & =\mu^{-2 \widetilde{N}_{c}-2} w^{2 \widetilde{N}_{c}+2-2 N_{f}}\left(w^{2}-w_{+}^{2}\right)^{r}\left(w^{2}-w_{-}^{2}\right)^{N_{f}-r}
\end{aligned}
$$

where $r=0,1, \ldots,\left[N_{f} / 2\right]$ and $w_{+}, w_{-}$are solutions of

$$
\begin{aligned}
& w_{+}+w_{-}=i \mu m, \\
& w_{+}^{N_{f}-r-\left(\widetilde{N}_{c}+1\right)} w_{-}^{r-\left(\widetilde{N}_{c}+1\right)}=(-1)^{\widetilde{N}_{c}+1} i^{N_{f}}\left(\mu \widetilde{\Lambda}_{N=2}\right)^{N_{f}-2\left(\widetilde{N}_{c}+1\right)} .
\end{aligned}
$$

At this stage, we put $\widetilde{N}_{c}=-1$. Then the relation among the dynamical scales is $\widetilde{\Lambda}_{N=2}=\widetilde{\Lambda}=\lambda \Lambda^{2}$, and the configuration is given by

$$
\begin{aligned}
v w & =\mu^{-1}\left(w^{2}+w_{+} w_{-}\right), \\
t & =w^{-2 N_{f}}\left(w^{2}-w_{+}^{2}\right)^{r}\left(w^{2}-w_{-}^{2}\right)^{N_{f}-r},
\end{aligned}
$$

where $r=0,1, \ldots,\left[N_{f} / 2\right]$ and

$$
w_{+}+w_{-}=i \mu m, \quad w_{+}^{N_{f}-r} w_{-}^{r}=\left(i \lambda \mu \Lambda^{2}\right)^{N_{f}} .
$$

Note that

$$
v^{2}+m^{2}=\mu^{-2} w^{-2}\left(w^{2}-w_{+}^{2}\right)\left(w^{2}-w_{-}^{2}\right) .
$$

From this and the equation (4.34), we see that the curve passes through the $A_{N_{f}-1}$ singularity at $v= \pm i m, t=x=0$ in such a way that there are $\mathrm{CP}^{1}$ components with multiplicity $1,2, \ldots, r-1, r, \ldots, r, r-1, \ldots, 2,1$ (the number of $r$ 's is $N_{f}-2 r+1$ ) in the resolved surface.

As far as $w_{ \pm} \neq 0$, these curves satisfy the following boundary conditions. The curve extends to infinity in two directions:

$$
\begin{aligned}
v & \sim \mu^{-1} w, t \sim 1, w \sim \infty \\
\text { and } \quad w & \sim 0, t \sim\left(\lambda \Lambda^{2}\right)^{-2 N_{F}} v^{2 N_{f}}, \quad v \sim \infty
\end{aligned}
$$

Also

$$
t=0 \text { implies } v= \pm i m \text {. }
$$


It is easy to identify all holomorphic curves satisfying this boundary condition. Those with a single infinite component are the curves given above and similar ones but with the second equation of (4.35) replaced by $w_{+}^{N_{f}-r} w_{-}^{r}=-\left(i \lambda \mu \Lambda^{2}\right)^{N_{f}}$. $\ddagger$

In addition to these, there is a solution consisting of two infinite components. It is given by

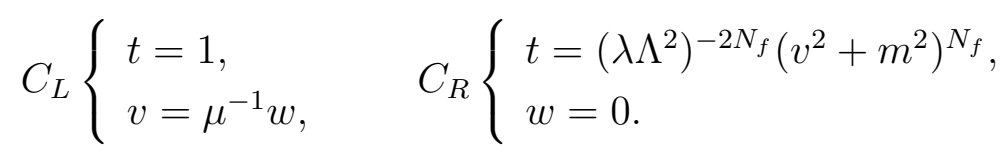

It is easy to see that this curve does satisfy the boundary condition given above. However, for generic values of $m$, the two components intersect the $\mathbf{Z}_{2}$ fixed plane $v=w=0$ at different points, $C_{L}$ at $t=1$ while $C_{R}$ at $t=\left(m / \lambda \Lambda^{2}\right)^{2 N_{f}}$ (see figure 5), and therefore the
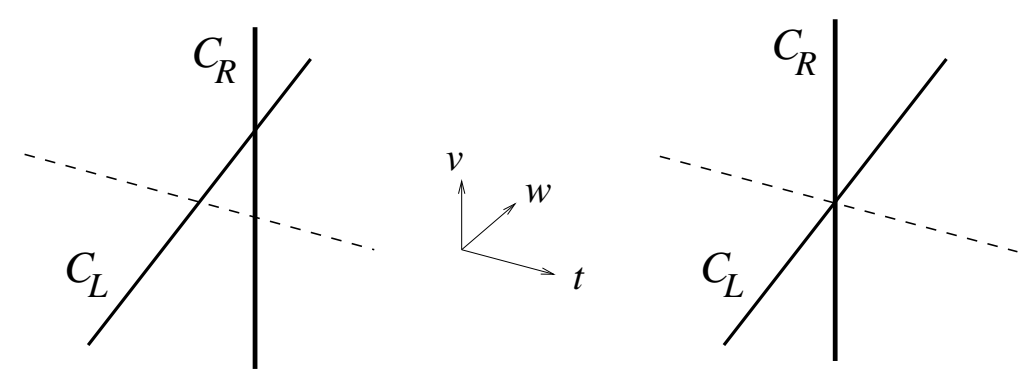

Figure 5: The configuration (4.40) near the $\mathbf{Z}_{2}$ fixed plane for $m^{N_{f}} \neq\left(\lambda \Lambda^{2}\right)^{N_{f}}$ (left) and $m^{N_{f}}=\left(\lambda \Lambda^{2}\right)^{N_{f}}$ (right). Note that the left one is a $t$-configuration.

configuration is not supersymmetric by the basic hypothesis. The two intersection points coincide only when $m^{N_{f}}=\left(\lambda \Lambda^{2}\right)^{N_{f}}$, and only then can the configuration be considered as defining a supersymmetric vacuum. Actually, in the case $m^{N_{f}}=\left(\lambda \Lambda^{2}\right)^{N_{f}}$, one of the $r=0$ solutions of (4.33)-(4.34) has $w_{-}=0$ and factorizes into two components, and is identical to the above solution.

As in [18], we can interpret $w_{ \pm}$as the eigenvalues of the matrix $S_{i j}$ by the R-symmetry and the flavor symmetry breaking pattern. Indeed, there is a precise correspondence of the values for each $r$ (compare (4.34) with (4.22) and (4.35) with (4.23)):

$$
\sigma_{ \pm}=i w_{ \pm}
$$

${ }^{\ddagger}$ The two types of solutions are related by a change of the sign of $\Lambda^{2 N_{f}}$. Thus, the presence of such copies is related to the doubling phenomena which we encountered before. In the present case, there is no obvious way to relate these curves by a coordinate change and it is not clear whether they can be considered as equivalent. However, as the presence of such copies does not make any change in the discussion of dynamical supersymmetry breaking, we simply ignore this doubling, by assuming that there is a way to show the equivalence to the curves (4.33)-(4.35). 
We also note that there are $r\left(N_{f}-r\right) \mathrm{CP}^{1}$ components at $v=i m$ together with their mirror images at $v=-i m$ and this is consistent with the fact that the complex dimension of the $r$-th branch is equal to $2 r\left(N_{f}-r\right)$. The extra solution present in the case $m^{N_{f}}=\left(\lambda \Lambda^{2}\right)^{N_{f}}$ can be identified with the supersymmetric vacuum with $S=0$.

\section{The Supersymmetric Configurations for $\mu=\infty$}

Let us take the $\mu \rightarrow \infty$ limit by keeping fixed $\lambda, \Lambda$ and $m$. The configuration with a single infinite component goes away from the region with finite $v w$ and becomes infinitely elongated in the $x^{6}$ direction since $w_{+}$and $w_{-}$diverge as $\sim \mu$ in the limit. The configuration with two infinite components present for $m^{N_{f}}=\left(\lambda \Lambda^{2}\right)^{N_{f}}$ has a limit given by

$$
C_{L}\left\{\begin{array} { l } 
{ t = 1 , } \\
{ v = 0 , }
\end{array} C _ { R } \left\{\begin{array}{l}
t=\left(\lambda \Lambda^{2}\right)^{-2 N_{f}}\left(v^{2}+m^{2}\right)^{N_{f}}, \\
w=0 .
\end{array}\right.\right.
$$

If we tune the parameter $m$ so that it approaches $\lambda \Lambda^{2}$ (or its $\mathbf{Z}_{N_{f}}$ phase rotation) as $m=\lambda \Lambda^{2}-\sigma / \mu$, one of the $r=0$ solutions is given by $w_{+}=i \mu \lambda \Lambda^{2}, w_{-}=-i \sigma$ and the $\mu \rightarrow \infty$ limit is a curve with a single component given by

$$
\left\{\begin{array}{l}
v w=\lambda \Lambda^{2} \sigma \\
t=\left(\lambda \Lambda^{2}\right)^{-2 N_{f}}\left(v^{2}+m^{2}\right)^{N_{f}} .
\end{array}\right.
$$

We note that (4.42) can be obtained from (4.43) by taking the limit $\sigma \rightarrow 0$. These supersymmetric configurations correspond to the supersymmetric vacua $S=\sigma J$ of the IYIT model with $m^{N_{f}}=\left(\lambda \Lambda^{2}\right)^{N_{f}}$.

\section{The Type IIA Limit}

We have proposed a realization of the (perturbed) IYIT model on the worldvolume of the fivebrane in $M$ theory. In the construction, we used a fictitious duality between $S p\left(N_{c}\right)$ and $S p(-1)$ "gauge" theories as a guide to read off the boundary condition. Although we have observed some quantitative and qualitative agreement with field theory about the supersymmetric ground states, it would be better to have more evidence for the proposal. One obvious thing to check is whether we can see the elementary fields of the gauge theory by considering the weak coupling Type IIA limit. In Type IIA string theory, the IYIT model (e.g. for $m=0$ ) may be realized as the worldvolume theory of the brane configuration as depicted in Figure 6. We can see the $S p\left(N_{c}\right)$ gauge symmetry on the D4branes stretched between the NS and $\mathrm{NS}^{\prime} 5$-branes, and the fundamental chiral multiplets are created by open strings ending on these D4-branes and the $2 N_{f}$ D4-branes ending on 


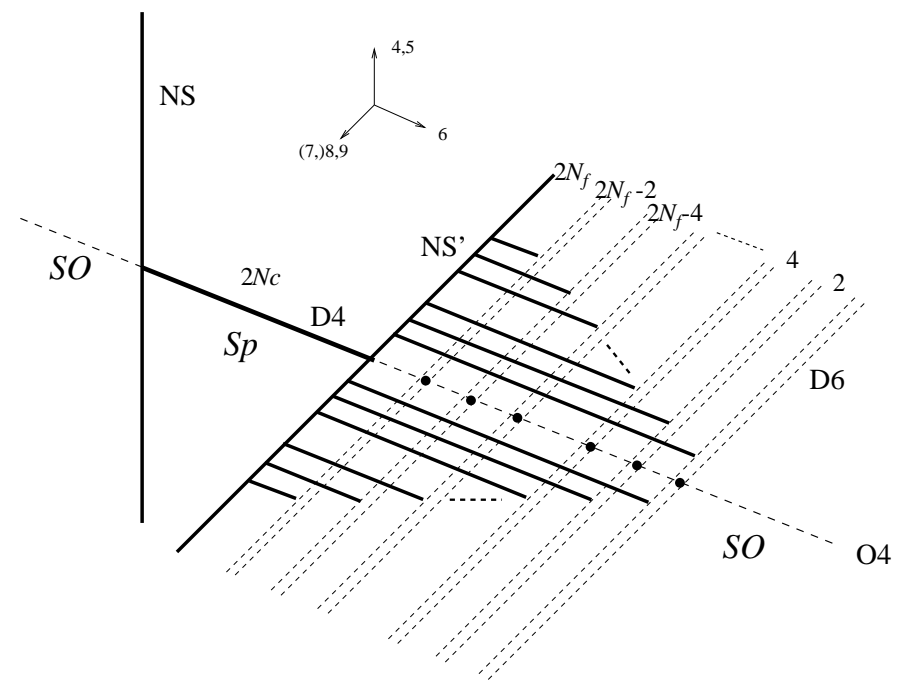

Figure 6: Type IIA Configuration for IYIT Model

the NS' brane from the right. The fluctuations of the singlet $S_{i j}$ correspond to the motion in the $x^{(7), 8,9}$ directions of the D4-branes on the right of the $\mathrm{NS}^{\prime} 5$-brane. By taking a suitable Type IIA limit of the above factorized configuration (which is a $t$-configuration for $m=0$ ), we can actually see this kind of intersecting brane configuration. See [68] for details.

\subsection{Non-supersymmetric Stable Vacuum}

Let us study the case with $m^{N_{f}} \neq\left(\lambda \Lambda^{2}\right)^{N_{f}}$ in detail. As we have seen above, in the $\mu \rightarrow \infty$ limit all supersymmetric configurations go away from the region with finite $v w$ and become infinitely elongated in more than four directions. We have already seen a similar phenomenon in the study of fivebrane configurations corresponding to $S U\left(N_{c}\right)$ SQCD with $N_{f}<N_{c}$ flavors in which there is no stable vacuum [18]. However, there is a clear difference that distinguishes the present case from such examples: The holomorphic curve (4.40) remains in the finite region and does not become infinitely elongated, although it does not define a supersymmetric vacuum because it contains a t-configuration. We discuss what this fact implies for the issue of existence of stable vacua.

As the classical Nambu-Goto action of the fivebrane shows, the area of the real twodimensional surface $\Sigma$ on which the fivebrane wraps plays the role of the potential energy. As far as the characteristic length scale of the brane is much larger than the elevendimensional Planck length, the classical supergravity is a good description and an area- 
minimizing surface can be considered as defining a stable vacuum as stated in section 2. Therefore one is tempted to look for an area-minimizing surface which satisfies the boundary condition given by (4.37)-(4.39) or its $\mu \rightarrow \infty$ limit. Of course, when the fivebrane approaches the $\mathbf{Z}_{2}$ fixed plane one has to take the effects of the fixed plane into account. The hypothesis that a $t$-configuration is not supersymmetric suggests that there is an extra potential energy for a $t$-configuration in addition to the energy associated with the area of the surface. In this paper, we assume that this energy is negligible compared to the energy coming from the area as long as the fivebrane is separated from the $\mathbf{Z}_{2}$ fixed plane by a distance much larger than the eleven-dimensional Planck length.

Although the surface we are considering is non-compact, one can define a regularized area as far as the surfaces obey a certain boundary condition at infinity. A formal expression of the area of a surface $\Sigma$ embedded in the seven-dimensional part of the space-time (transverse to the $\mathbf{R}^{4}$ direction which the four-dimensional part of the fivebrane spans) is given by

$$
\operatorname{Area}(\Sigma)=\int_{\Sigma} \sqrt{g} \mathrm{~d}^{2} x
$$

where $\sqrt{g} \mathrm{~d}^{2} x$ is the area element of the metric $g_{\mu \nu}$ on the surface induced from the sevenmanifold. In the present case, the seven manifold is the product of the Taub-NUT space parametrized by $(t, v)$ and $\mathbf{R}^{3}$ parametrized by $\left(w^{7}, w=w^{8}+i w^{9}\right)$. Since we are interested in area-minimizing surfaces under the condition that $w^{7} \rightarrow 0$ in each asymptotic region, we may as well consider only the surfaces with $w^{7} \equiv 0$. Namely, we consider $\Sigma$ to be a surface embedded in the direct product of the Taub-NUT space and the flat $w$-plane which is a manifold with a Kähler metric $G_{i \bar{\jmath}}$ where we use $i, j(\bar{\imath}, \bar{\jmath})$ to denote the indices of local (anti-)holomorphic coordinates. The induced metric $g_{\mu \nu}$ defines a complex structure on $\Sigma$. Then, the area element can be expressed as

$$
\begin{aligned}
\sqrt{g} \mathrm{~d}^{2} x & =g_{z \bar{z}} \mathrm{~d}^{2} z=G_{i \bar{\jmath}}\left(\partial_{z} X^{i} \partial_{\bar{z}} X^{\bar{\jmath}}+\partial_{\bar{z}} X^{i} \partial_{z} X^{\bar{\jmath}}\right) \mathrm{d}^{2} z \\
& =2 G_{i \bar{\jmath}} \partial_{\bar{z}} X^{i} \partial_{z} X^{\bar{\jmath}} \mathrm{d}^{2} z+\frac{i}{2} G_{i \bar{\jmath}} \mathrm{d} X^{i} \wedge \mathrm{d} X^{\bar{\jmath}},
\end{aligned}
$$

where $z$ is a local complex coordinate of $\Sigma$. The integral of the first term is finite if the boundary condition at infinity is holomorphic and if the deviation $\partial_{\bar{z}} X^{i}$ from the holomorphic embedding falls off sufficiently fast. To put it more appropriately, we include the finiteness of the integral of this term as one of the boundary conditions. The second term of (4.45) is the Kähler form of the space-time restricted to the surface $\Sigma$. Since the Kähler form is a closed two-form, its integral does not change for a continuous variation of the surface and we may consider it as a constant. Thus, we may as well take

$$
\operatorname{Area}^{\prime}(\Sigma)=2 \int_{\Sigma} G_{i \bar{\jmath}} \partial_{\bar{z}} X^{i} \partial_{z} X^{\bar{\jmath}} \mathrm{d}^{2} z
$$


as the definition of the area as long as we are considering surfaces in a given homology class.

Actually, the integral of the second term of (4.45) is divergent and needs some special care in order to show that it can really be considered as a constant. We first regularize the integral by cutting off some part of the surface at infinity. In general, a variation of the surface induces a change in the boundary of the cut-off surface, and the integral might change by a boundary term. The integral can be considered as a constant only when the boundary term vanishes as we take the limit where the cut-off is removed. For the configurations we are studying there is a certain regularization such that the boundary term does vanish as we remove the cut-off, as we now show in the $\mu=\infty$ case (the generalization to the case with finite $\mu$ is obvious). In the asymptotic region (4.37) where the surface is parametrized by $w$, we cut off the part with $|w|>W$ for some large $W$. Similarly we cut off $|v|>V$ in the other asymptotic region (4.38) for some large $V$. It is easy to see that the boundary term vanishes in the $V, W \rightarrow \infty$ limit. For example, let us consider the boundary term in the asymptotic region (4.37). In the region with large $w$, the surface is considered as a graph of the functions $v(w), t(w)$, and the variation $\Sigma_{0} \rightarrow \Sigma_{1}$ of the surface is represented by the variation of such functions $v_{0}(w), t_{0}(w) \rightarrow v_{1}(w), t_{1}(w)$. The boundary term is the integral of the Kähler form on the boundary surface at $|w|=W$. As the boundary surface, one can take for example $w=W \mathrm{e}^{i \sigma}, v=v_{0}(w)+\tau\left(v_{1}(w)-v_{0}(w)\right), t=t_{0}(w)+\tau\left(t_{1}(w)-t_{0}(w)\right)$ parametrized by $0 \leq \sigma<2 \pi, 0 \leq \tau \leq 1$. Since the Kähler form is the sum of $i \mathrm{~d} w \wedge \mathrm{d} \bar{w}$ and the Kähler form of the Taub-NUT space which is well-parametrized by $v$ and $t$ near $t=1$ and $v=0$, the boundary term vanishes in the limit $W \rightarrow \infty$ as long as $\left|v_{1}(w)-v_{0}(w)\right|,\left|t_{1}(w)-t_{0}(w)\right| \rightarrow 0$ which hold under the boundary conditions (4.37). Thus, one can really take (4.46) as the definition of the regularized area.

The basic property of the regularized area (4.46) is that it vanishes only for holomorphic curves. Therefore, if there is a holomorphic curve satisfying the boundary condition, an area minimizing surface $\Sigma$ in the same homology class must be holomorphic since the holomorphic curve has Area' $=0$ and hence an area minimizing surface must also have Area $^{\prime}=0$ which implies that it is holomorphic.

For finite $\mu$, there are various holomorphic curves satisfying the boundary condition. All these including the factorized one (4.40) are connected by a continuous deformation, as can be seen as follows. Since the space-time is topologically trivial in the $w$ direction, we only have to show that their projections to the Taub-NUT space are connected by a deformation. The projection of the curve (4.33)-(4.34) at the $r$-th branch, including the 
$\mathrm{CP}^{1}$ components, is described by the equation

$$
x+y=\left(\lambda \Lambda^{2}\right)^{-2 N_{f}}\left(v^{2}+m^{2}\right)^{N_{f}}+u_{1}\left(v^{2}+m^{2}\right)^{N_{f}-1}+\cdots+u_{N_{f}-r}\left(v^{2}+m^{2}\right)^{r},
$$

for certain $u_{i}$ 's, while the factorized curve (4.40) projects to

$$
x+y=\left(\lambda \Lambda^{2}\right)^{-2 N_{f}}\left(v^{2}+m^{2}\right)^{N_{f}}+1 .
$$

It is evident that these are connected by a continuous deformation that does not change the asymptotic behavior. It is not clear whether this implies that we should only take into account the surfaces in the same homology class, but we shall assume this to be the case. Then, all the holomorphic curves have the same minimum area Area' $=0$ and all other surfaces have Area' $>0$. The graph of the area functional is thus schematically given by figure 7 .

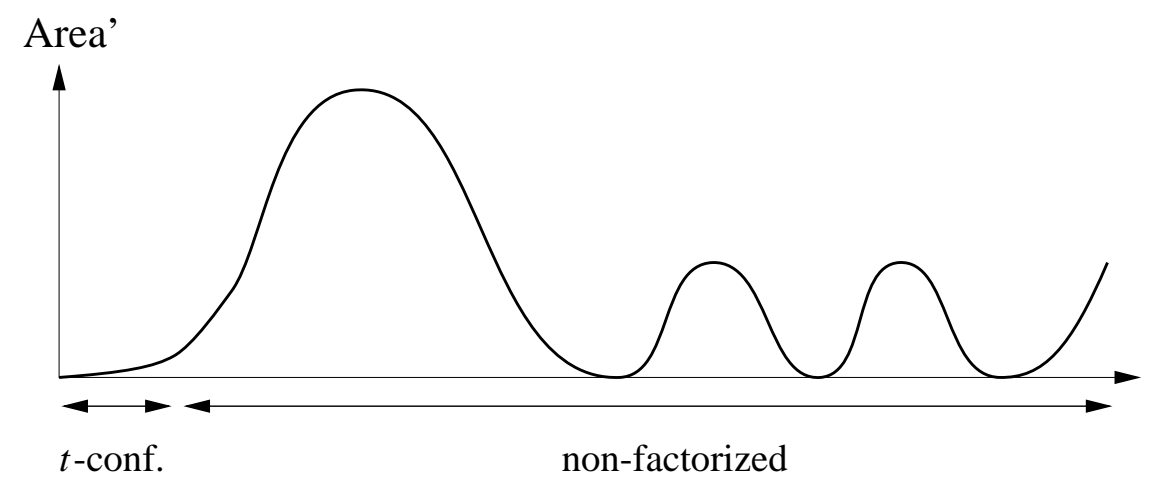

Figure 7: The Graph of Area'

The horizontal axis parametrizes the space of surfaces. In the region called "t-conf.", the surface contains a $t$-configuration while the surface does not intersect the $\mathbf{Z}_{2}$ fixed plane in the region called "non-factorized". In the transition region between them, two parts of the surface intersect the fixed plane at the same point. The minima correspond to the holomorphic curves. The minima with non-factorized curves run away to infinity in the $\mu \rightarrow \infty$ limit, but the minimum in the "t-conf." region is the factorized curve (4.40) and does not go away.

Our basic hypothesis that a $t$-configuration is not supersymmetric means that the configuration with factorized holomorphic curve has positive energy compared to the non-factorized holomorphic curves, although it minimizes the area. More generally, such an extra energy should be positive in the whole "t-conf." region, fall down toward the transition region, and be negligible in the part of the "non-factorized" region in which 
the curve is separated from the $\mathbf{Z}_{2}$ fixed plane by a distance much larger than the elevendimensional Planck scale. The competition between the force from this extra energy and the force from the gradient of Area' determine the location of a possible stable configuration.

Therefore, it is important to know how fast the area grows as the factorized curve (4.40) is deformed so that the two intersection points approach each other. Namely, we want to find an area minimizing surface with a given distance between the two components and compute the area as a function of the distance. For simplicity let us consider the $\mu=\infty$ case. The component $C_{L}$ is at $t=1, v=0$ and intersects the $\mathbf{Z}_{2}$ fixed plane $v=w=0$ at $t=1$ while the component $C_{R}$ is at $t=\left(\lambda \Lambda^{2}\right)^{-2 N_{f}}\left(v^{2}+m^{2}\right)^{N_{f}}, w=0$ and intersects the $\mathbf{Z}_{2}$ fixed plane at $t=\left(m / \lambda \Lambda^{2}\right)^{2 N_{f}}$. We want for example to deform $C_{L}$ by replacing $t \equiv 1$ by $t=t(w)$, a function of $w$, so that the area is minimized under the condition $t(w) \rightarrow 1$ as $w \rightarrow \infty$ and $t(0)=1+\Delta$ for a fixed $\Delta$. One can consider an analogous problem in the simplified one-dimensional analog where the parameters $t, w$ are considered as real numbers and minimum area is replaced by minimum length. In this one-dimensional analog, for the configuration given by $t(w) \equiv 1$ for $|w| \geq \epsilon$, and $t(w)=1+\Delta(1-|w| / \epsilon)$ for $|w| \leq \epsilon$ (see figure 8), the length decreases as $\epsilon$ is increased and does not attain a minimum as the $\epsilon \rightarrow \infty$ limit no longer satisfies the required asymptotic behavior.

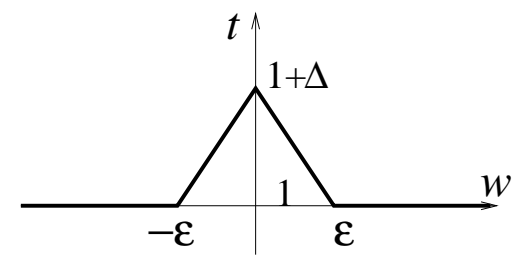

Figure 8: A One-Dimensional Analog

One may wonder whether our two-dimensional model exhibits this "run-away" behavior. To examine this, we compute the area of the surface in the Euclidean space $\mathbf{C}^{2}$ given by the same function $t(w)$ as above where now we consider $t, w$ to be complex variables. One may be worried by the fact that the surface is not smooth at $|w|=0$ and $\epsilon$ because a singularity might suggest an instability of the configuration, but such a singularity can be smoothed out without change of the area. The difference of the area from the one of the flat surface is given by

$$
\Delta \text { Area }=\left(\sqrt{1+\Delta^{2} / \epsilon^{2}}-1\right) \pi \epsilon^{2} .
$$


This is positive and is monotonically increasing as a function of $\epsilon$. Therefore, in the twodimensional case, we may not have to worry about such a run-away behavior for $\epsilon \rightarrow \infty$ as in the case of one-dimensional analog.

However, there is another problem. The difference $\Delta$ Area (4.49) decreases as we decrease $\epsilon$ and approaches zero as $\epsilon \rightarrow 0$ but the $\epsilon=0$ surface has a spike singularity which cannot be smoothed out without increasing the area. Namely, for any fixed $\Delta$, the area can be made arbitrarily small but no smooth surface can attain Area $^{\prime}=0$. In particular, this is also true for the case $\Delta=\left(m / \lambda \Lambda^{2}\right)^{2 N_{f}}-1:=\Delta_{*}$ in which the two intersection points coincide. However, we cannot conclude from this that there is no local minimum of the energy. This is because a surface with sufficiently small Area' has characteristic length $\epsilon$ smaller than the eleven-dimensional Planck length, and our argument (based on the low energy action) does not apply to such surfaces. Precisely because of this reason, we cannot make a decisive statement concerning the stable vacuum within the eleven-dimensional supergravity approximation of $M$ theory.

We have found that the slope of Area' is almost zero as a function of the distance between the two intersection points. On the other hand, we expect a rapid growth of the extra energy as the two intersection points are separated: Otherwise, there would be an extra light mode in the examples we have considered in the previous section which is absent in the corresponding $S p\left(N_{c}\right)$ gauge theories. Therefore, it is unlikely that the potential minimum is in the "t-conf." region, although we need a more careful estimate of the growth of the extra energy in order to completely exclude this possibility.

The fact that $\Delta$ Area (4.49) approaches zero when approaching the surface with a spike singularity shows that Area' in the "non-factorized" region also falls off when approaching the spike surface with $\Delta=\Delta_{*}$. In other words, Area' grows when going away from the spike surface in the direction to the "non-factorized" region. In order to illustrate this, we consider a family of configurations parametrized by $s$ in the $\mu=\infty$ case as depicted in figure $9 .^{1}$ As $s \rightarrow 0$, the configurations approach the surface with the spike singularity. To be more precise, we construct the configurations from the factorized curve (4.40) by cutting off the two discs - $|w| \leq s$ of $C_{L}$ and $|v| \leq s$ of $C_{R}$ - and connect the boundary circles by a cylinder (for simplicity of the discussion, we assume that $v, w$ and $t$ are coordinates of the flat Euclidean space $\mathbf{C}^{3}$ with the metric given by $\left.|\mathrm{d} v|^{2}+|\mathrm{d} w|^{2}+|\mathrm{d} t|^{2}\right)$. As the cylinder, we can choose $t=1+\Delta_{*} \tau, v=s f(\tau) \mathrm{e}^{i \theta}$ and $w=s g(\tau) \mathrm{e}^{-i \theta}$, where $0 \leq \tau \leq 1,0 \leq \theta \leq 2 \pi$ are the coordinates of the cylinder, and $f(\tau)$ (resp. $g(\tau)$ ) is some non-negative function starting from $f(0)=0$ and ending at $f(1)=1$ (resp. from $g(0)=1$

\footnotetext{
${ }^{1}$ We thank Michael Peskin for asking a question which lead to this computation.
} 


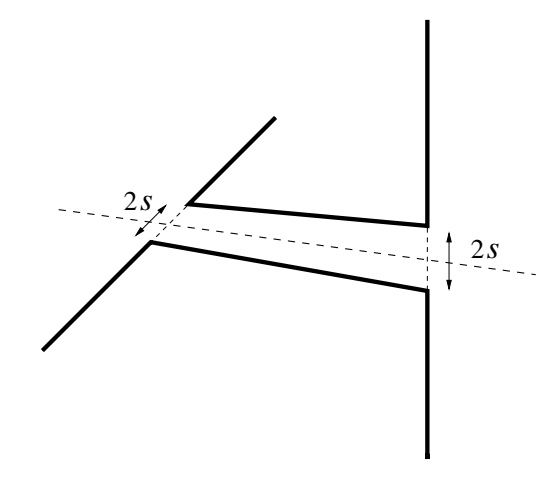

Figure 9: A Deformation From The Spike Surface

to $g(1)=0)$ such that $f^{2}+g^{2}$ is always positive. Then the Area' is given by

$$
\text { Area }^{\prime}=2 \pi s^{2} \int_{0}^{1} \sqrt{\Delta_{*}^{2} / s^{2}+\left(f^{\prime}\right)^{2}+\left(g^{\prime}\right)^{2}} \sqrt{f^{2}+g^{2}} \mathrm{~d} \tau-2 \pi s^{2} .
$$

One can show by using the inequality $\sqrt{\left(f^{\prime}\right)^{2}+\left(g^{\prime}\right)^{2}} \sqrt{f^{2}+g^{2}} \geq f f^{\prime}-g g^{\prime}$ that this is indeed non-negative. Since it is zero at $s=0$, it can never decrease as $s$ is increased. One can actually show using the same inequality that it is monotonically increasing as a function of $s$ at all values of $s \geq 0$ for an arbitrary choice of the differentiable functions $f(\tau), g(\tau)$. The parameter $s$ can be considered as a counterpart of the eigenvalue $\sigma$ of the singlet $S_{i j}$, as the supersymmetric configuration (4.43) in the $\Delta_{*}=0$ case suggests. The monotonic growth of Area' as $s \rightarrow$ large may be considered as an analog of the potential growth as $\sigma \rightarrow$ large in the field theory which can be shown near the ultra-violet cut-off $\lambda \sigma \lesssim M_{\mathrm{UV}}$ by the one loop computation (and can be continued to smaller values of $\sigma$ to some extent by a renormalization group argument). In the brane picture the Area' itself continues to fall off as $s \rightarrow$ small up until $s=0$. Of course, Area' can really be considered as the potential energy only if the length scale set by $s$ is much larger than the eleven-dimensional Planck length.

For finite $\mu$, Area' also decreases when approaching any of the non-factorized holomorphic curves, but these go away to infinity in the $\mu \rightarrow \infty$ limit, while the fall-off toward the spike surface remains as we have illustrated above. In addition, there are no other obvious directions in which the potential decreases. This may suggest that, in the limit $\mu \rightarrow \infty$, there is a minimum of the potential energy in a region near the singular surface. In field theory, it was difficult to obtain definite information about the stable vacuum, such as its location or vacuum energy, because of the difficulty in analyzing the Kähler potential of $\sigma$ in the region where $\sigma$ is small. In the brane picture, the difficulty is in 
dealing with the fivebrane which has a characteristic length scale smaller than the Planck length, for which we need information about the fivebrane dynamics in $M$ theory beyond the eleven-dimensional supergravity approximation.

\section{Remark}

In [69], the IYIT model was realized in $S O(32)$ heterotic string theory compactified on a K3-fibred Calabi-Yau three-fold by encoding the $S U(2)$ gauge symmetry in a singularity of the gauge bundle along a section (a Riemann surface) of the K3-fibration. It would be interesting to find a relation with our construction of the model. However, there is a clear difference from ours. The parameter corresponding to the dynamical scale $\Lambda$ is related by $\sqrt{\alpha^{\prime}} \Lambda=\mathrm{e}^{-R^{2} / \alpha^{\prime}}$ to the size $R$ of the Riemann surface, which is one of the moduli of string theory. Since the vacuum energy would be $\Lambda^{4}$ times some dimensionless quantity, this may cause a problem of vacuum instability. In our case, it takes an infinite amount of energy to vary the parameter $\Lambda$ because a change of $\Lambda$ causes a change of the asymptotic boundary condition. Therefore, a map between our model and that of [69], if exists, would be realized in some limit which freezes the degrees of freedom corresponding to the variation of the size of the Riemann surface on the heterotic side.

\section{$5 \quad S p \times S O$ - Run away Behavior}

In this section we will study the IYIT model with a gauged flavor group. This theory has several features in common with the original IYIT model, but there is one crucial difference. In the case where $N_{f}=N_{c}+1$, supersymmetry is still broken, but there no longer exists a stable non-supersymmetric vacuum. It is therefore very interesting to compare the brane geometry for the gauged model to the one for the original IYIT model discussed in the previous section. It may provide us with a general rule how to distinguish between runaway behavior and the existence of stable non-supersymmetric vacua, given some brane configuration for a theory with dynamical supersymmetry braking.

\section{$5.1 \quad$ Field Theory}

One way to construct a brane configuration for an $N=1$ theory is to start with an $N=2$ theory and to introduce suitable mass terms so that when we send the masses to infinity we recover the $N=1$ theory. We used this in the previous sections to study $N=2$ QCD with gauge group $S p\left(N_{c}\right)$ as well as the IYIT model. The advantage of 
this method is that one can see which $N=1$ theories have a supersymmetric vacuum and which ones do not. If the brane configuration has a well-defined limit when we send the masses to infinity, then this is the brane configuration for the corresponding $N=1$ theory. If no such well-defined limit exists, supersymmetry is generically broken in the $N=1$ theory. Clearly, this argument is only valid if supersymmetry is unbroken for finite values of the masses. To facilitate comparison with the brane geometry, we will therefore discuss an $N=2$ version of the gauged IYIT model, broken to $N=1$ by mass terms.

We thus consider an $N=2$ gauge theory with gauge group $S p\left(N_{c}\right) \times S O\left(2 N_{f}\right)$, and one hypermultiplet $Q_{a i}$ transforming in the 'bifundamental' representation $\left(\mathbf{2 N}_{\mathbf{c}}, \mathbf{2 N}_{\mathbf{f}}\right)$. From the $N=1$ point of view there are two chiral superfields $\left(\Phi_{\mathrm{Sp}}\right)_{a b},\left(\Phi_{\mathrm{SO}}\right)_{i j}$ transforming in the adjoint representations of $S p\left(N_{c}\right)$ and $S O\left(2 N_{f}\right)$ respectively. The superpotential, including a mass term for $\Phi_{\mathrm{Sp}}$, reads

$$
W=\operatorname{Tr}\left(Q^{t} J \Phi_{\mathrm{Sp}} J Q\right)+\operatorname{Tr}\left(J Q \Phi_{\mathrm{SO}} Q^{t}\right)+\mu \operatorname{Tr}\left(J \Phi_{\mathrm{Sp}} J \Phi_{\mathrm{Sp}}\right) .
$$

Under gauge transformations $Q \rightarrow g Q h^{t}, \Phi_{\mathrm{SO}} \rightarrow h \Phi_{\mathrm{SO}} h^{t}$ and $\Phi_{\mathrm{Sp}} \rightarrow g \Phi_{\mathrm{Sp}} g^{t}$, where $g \in S p\left(N_{c}\right)$ and $h \in S O\left(2 N_{f}\right)$. For large $\mu$ we can integrate out $\Phi_{\mathrm{Sp}}$. Introducing the meson field $M=Q^{t} J Q$, the resulting superpotential after integration reads

$$
W=-\frac{1}{4 \mu} \operatorname{Tr}(M M)+\operatorname{Tr}\left(M \Phi_{\mathrm{SO}}\right) .
$$

To analyze what happens in the quantum theory it is convenient to know the charges of the various fields and parameters under the global $U(1)_{A} \times U(1)_{R}$ symmetry. They are given by

$$
\begin{array}{cccccc} 
& Q & \Lambda_{N=1, S p}^{3\left(N_{c}+1\right)-N_{f}} & \mu & \Phi_{\mathrm{SO}} & \Lambda_{N=2, S O} \\
U(1)_{A} & 1 & 2 N_{f} & 4 & -2 & -2 \\
U(1)_{R} & 1-\frac{N_{c}+1}{N_{f}} & 0 & 2-4 \frac{N_{c}+1}{N_{f}} & 2 \frac{N_{c}+1}{N_{f}} & 2 \frac{N_{c}+1}{N_{f}} .
\end{array}
$$

The $U(1)_{R}$ charges have been chosen so that they yield the usual $U(1)_{R}$ charge assignments for the $S p\left(N_{c}\right)$ theory.

We now discuss the four different cases $N_{f} \leq N_{c}, N_{f}=N_{c}+1, N_{f}=N_{c}+2$ and $N_{f}>N_{c}+2$.

$N_{f} \leq N_{c}$. The classical moduli space is given by $M=0$ while $\Phi_{\mathrm{SO}}$ takes values in the Cartan subalgebra. In other words, it is just the moduli space of the $N=2 S O\left(2 n_{f}\right)$ theory. In the quantum theory, strong coupling dynamics can generate corrections to the superpotential. The global symmetry restricts the form of such a correction to a flavor invariant combination of the form

$$
W \sim \mu^{-\alpha}\left(\frac{\Lambda_{N=1, S p}^{3\left(N_{c}+1\right)-N_{f}}}{M^{N_{f}}}\right)^{\beta /\left(N_{c}+1-N_{f}\right)} \Lambda_{N=2, S O}^{\gamma} M^{1-\beta+\alpha} \Phi_{\mathrm{SO}}^{1-\alpha-\beta-\gamma} .
$$


If all the nonperturbative dynamics is due to instanton effects then $\beta$ and $\gamma$ must be nonnegative integers. Because of holomorphy, $\alpha$ must also be a nonnegative integer. The dynamics of the $S O\left(2 N_{f}\right)$ gauge group does not generate any non-perturbative superpotential because from that point of view the theory looks like an $N=2$ theory. The dynamics of the $S P\left(N_{c}\right)$ theory is that of an $N=1$ theory with massive quarks. In such a theory a non-perturbative superpotential is generated, which is given by [52],

$$
W_{d y n} \sim\left(\frac{\Lambda_{N=1, S p}^{3\left(N_{c}+1\right)-N_{f}}}{\operatorname{Pf} M}\right)^{1 /\left(N_{c}+1-N_{f}\right)} .
$$

The full superpotential $W$ is the sum of (5.2) and (5.5).

An alternative derivation of the superpotential is obtained by putting a coefficient $\lambda$ in front of the second term in (5.2). For $\lambda \rightarrow 0$ the theory is well-behaved and the full superpotential should therefore be analytic in $\lambda$. This imposes the restriction $\alpha+\beta+\gamma \leq 1$ in (5.4). The term with $\gamma=1, \alpha=\beta=0$ cannot be present as it cannot be made invariant under $S O\left(2 N_{f}\right)$. The remaining possibilities correspond to the three terms in $W=(5.2)+(5.5)$.

The full superpotential implies that supersymmetry is dynamically broken, as the $\partial W / \partial \Phi_{\mathrm{SO}}=M=0$ equation is incompatible with the equation $\partial W / \partial M=0$ due to (5.5). We will later see a confirmation of this in the brane analysis. A similar situation appears in the case of $S U$ gauge groups, see [36].

$N_{f}=N_{c}+1$. For this value of $N_{f}$, global symmetries restrict a contribution to the superpotential to be of the form

$$
\mu^{-\alpha} \Lambda_{N=1, S p}^{2 \beta} \Lambda_{N=2, S O}^{\gamma} M^{1+\alpha-\beta} \Phi_{\mathrm{SO}}^{1-\alpha-\gamma} .
$$

By going to large $\Phi_{\mathrm{SO}}$ we can see that none of the possible terms will be generated, as the only dynamics is that of a massive $N=1 S P\left(N_{c}\right)$, plus that of some $U(1) N=2$ vector multiplets. The only nontrivial dynamics comes from the $S p\left(N_{c}\right)$ gauge group, which generates the quantum constraint $\operatorname{Pf} M=\Lambda_{N=1, S p}^{2 N_{f}}$. This is incompatible with the equation $M=0$ obtained from the tree level superpotential (5.2), and supersymmetry is dynamically broken. To analyze whether there is a stable vacuum or not, we focus in analogy to section four on the region where $\Phi_{S O}$ is large. In that region the quarks acquire a large mass and can therefore be integrated out. What remains is a pure $S p\left(N_{c}\right)$ gauge theory with a scale $\Lambda_{N=1}$, together with an $S O\left(2 N_{f}\right)$ gauge theory with the adjoint matter field $\Phi_{S O}$. Gaugino condensation in the pure $S p\left(N_{c}\right)$ gauge theory generates a superpotential $W \sim \Lambda_{N=1}^{3}$. Using the scale matching relation $\Lambda_{N=1}^{3\left(N_{c}+1\right)}=\operatorname{Pf}\left(\Phi_{\mathrm{SO}}\right) \Lambda_{N=1, S p}^{3\left(N_{c}+1\right)-N_{f}}$ we can 
write this as

$$
W \sim\left(\operatorname{Pf}\left(\Phi_{\mathrm{SO}}\right)\right)^{1 / N_{f}} \Lambda_{N=1, S p}^{2}
$$

The scalar potential is given by $V \sim\left|\frac{\partial W}{\partial \Phi_{\mathrm{SO}}}\right|^{2} g^{\Phi_{\mathrm{SO}} \bar{\Phi}_{\mathrm{SO}}}$, where $g^{\Phi_{\mathrm{SO}} \bar{\Phi}_{\mathrm{SO}}}$ is the inverse of the metric appearing in the kinetic term for $\Phi_{\mathrm{SO}}$. Since $\partial W / \partial \Phi_{\mathrm{SO}}$ is roughly of order one, the behavior of the scalar potential for large $\Phi_{\mathrm{SO}}$ is determined by the behavior of $g_{\Phi_{\mathrm{SO}} \bar{\Phi}_{\mathrm{SO}}}$ for large $\Phi_{\mathrm{SO}}$.. This can be estimated using a one-loop calculation. The result of the one-loop calculation reads

$$
g_{\Phi_{\mathrm{SO}} \bar{\Phi}_{\mathrm{SO}}} \sim 1+\frac{1}{8 \pi^{2}}\left(2\left(2 N_{f}-2\right)-2 N_{c}\right) \log \left|\frac{\Phi_{\mathrm{SO}}}{M_{U V}}\right|
$$

with $M_{U V}$ the ultra-violet cut off. For large $\left|\Phi_{\mathrm{SO}}\right|$ we see that the metric becomes very large, and therefore the scalar potential has a runaway behavior and approaches zero at infinity (a similar behavior also occurs for $N_{f}<N_{c}+1$ ). This fact shows that the true minimum of the scalar potential is at infinity, and that there is no stable vacuum for large $\left|\Phi_{\mathrm{SO}}\right|$. This argument does not exclude the possibility of a local minimum in the scalar potential, but so far no evidence for the existence of such local minima has been found. The brane analysis will in fact provide evidence to the contrary.

$N_{f}=N_{c}+2$. The full superpotential is given by $W=(5.2)+(5.5)$. In the quantum theory $M$ is an unconstrained field that can take on any value [52]. The tree-level superpotential enforces $M=0$. Now, this point is compatible with (5.5) and part of the moduli space, it is the special point for the $S P\left(N_{c}\right)$ theory where there is confinement without chiral symmetry breaking. From the point of view of the $S O$ gauge theory, both $\Phi$ and $M$ are massive, because the tree-level superpotential equals $-\frac{1}{4 \mu} \operatorname{Tr}\left(M-2 \mu \Phi_{S O}\right)^{2}+\mu \operatorname{Tr} \Phi_{S O}^{2}$. Integrating them out leaves us with a pure $S O\left(2 N_{f}\right)$ gauge theory which has $2 N_{f}-2$ discrete vacua. Thus, the total number of discrete vacua is also $2 N_{f}-2=2\left(N_{c}+1\right)$.

$N_{f}>N_{c}+2$. At low energies the dynamics in the $S p$ gauge group is described by the dual magnetic theory with gauge group $S p\left(N_{f}-N_{c}-2\right)$ [66]. Using the dual description the tree-level superpotential reads

$$
W_{t r}=\frac{1}{4 \mu} \operatorname{Tr}(M M)+\frac{1}{\lambda} \operatorname{Tr}\left(J q M q^{t}\right)+\operatorname{Tr}\left(M \Phi_{S O}\right),
$$

where $q$ are the dual magnetic quarks and $\lambda$ is an extra scale that appears for dimensional reasons. The $S P\left(N_{f}-N_{c}-2\right)$ gauge group generates a nonperturbative superpotential $W_{d y}$ which is again given by (5.5). The tree-level superpotential forces the theory in the non-abelian Coulomb point $M=0$. At this point, the $S O$ part of the theory is an $N=1 S O\left(2 N_{f}\right)$ gauge theory with $2\left(N_{f}-N_{c}-2\right)$ massless flavors, because $\Phi_{\text {So }}$ and $M$ are massive, but the magnetic quarks $q$ are massless. This $S O\left(2 N_{f}\right)$ theory generates 
a non-perturbative superpotential due to gaugino condensation [67], and we see that supersymmetry is dynamically broken.

\subsection{Branes}

To construct a brane configuration for the gauge theory with gauge group $\operatorname{Sp}\left(N_{c}\right) \times$ $S O\left(2 N_{f}\right)$ and superpotential (5.1), we first construct the brane configuration for the $N=2$ gauge theory with $\mu=0$ and then try to introduce a mass term as in [18] by rotating one of the branes. The type IIA and M-theory brane construction for the $N=2$ gauge theory were discussed in [15]. The type IIA construction consists of 3 NS 5-branes, with $2 N_{c}$ D 4 branes stretched between the first and second fivebrane, and $2 N_{f}$ D 4 branes stretched between the second and the third. In addition, there is an $\mathrm{O} 4$ orientifold plane parallel to the D4 branes. With a suitable choice of sign for the orientifold projection this describes precisely the $N=2$ gauge theory of interest. After lifting the description to M-theory, the corresponding fivebrane configuration is given by the equation

$$
F(t, v) \equiv \Lambda_{N=2, S p}^{2\left(2 N_{c}+2-N_{f}\right)} t^{3}-\left(v^{2} P_{N_{c}}\left(v^{2}\right)+c\right) t^{2}+Q_{N_{f}}\left(v^{2}\right) t-v^{2} \Lambda_{N=2, S O}^{2\left(2 N_{f}-2-N_{c}\right)}=0,
$$

where $c$ is a constant (which can be determined by requiring that (5.10) is not a $t$ configuration) given by

$$
c^{2}=4 \Lambda_{N=2, S p}^{2\left(2 N_{c}+2-N_{f}\right)} Q(0)
$$

and $P_{N_{c}}$ and $Q_{N_{f}}$ are polynomials of order $N_{c}$ and $N_{f}$ in $v^{2}$. In the limit where $\Lambda_{N=2, S O} \rightarrow$ 0 , the curve becomes a double cover of the curve for $S p\left(N_{c}\right)$ gauge groups with matter given in [54], and similarly for $\Lambda_{N=2, S p} \rightarrow 0$ we recover the curve for $S O\left(2 N_{f}\right)$.

Recall that $v=x^{4}+i x^{5}$ and that rotation of a curve means that we rotate one of the NS fivebranes in the $w=x^{8}+i x^{9}$ direction. The curve (5.10) has three asymptotic regions where $v \rightarrow \infty$ and $t \sim v^{2 N_{c}+2}, t \sim v^{2 N_{f}-2 N_{c}-2}$ and $t \sim v^{2-2 N_{f}}$ respectively, corresponding to the three NS fivebranes. To give a mass to the adjoint $S p\left(N_{c}\right)$ superfield as in (5.1), we have to rotate the leftmost NS fivebrane (i.e. the one where $t \sim v^{2 N_{c}+2}$ ). Rotating the middle NS fivebrane corresponds to giving masses to both $\Phi_{\mathrm{Sp}}$ and $\Phi_{\mathrm{SO}}$, and rotating the rightmost NS fivebrane corresponds to giving a mass to $\Phi_{\mathrm{SO}}$ only.

Because $w$ and $\mu$ are the only objects that transform nontrivial under $U(1)$ rotations in the 8, 9 plane, we know that the projection of the rotated curve in the $t, v$ plane must be given by the original curve itself. Therefore, the rotated curve is given by the two equations $F(t, v)=0$ and $w=w(t, v)$, and the goal is to determine $w(t, v)$. If we compactify the original curve then $w$ would seem to be a meromorphic function on this compactified curve with a double pole the location of the leftmost NS fivebrane. However, we have 
to be more careful, because the space-time geometry is non-trivial due to the presence of the $\mathrm{O} 4$ plane. The $\mathbf{Z}_{2}$ action induced by the $\mathrm{O} 4$ plane maps $v \rightarrow-v$ and $w \rightarrow-w$. Modding out by this $\mathbf{Z}_{2}$ creates an $A_{1}$ singularity in the $v, w$ space, and $v, w$ are not good coordinates on the quotient. Therefore, we introduce the $\mathbf{Z}_{2}$ invariant coordinates

$$
p=v^{2}, \quad q=w^{2}, \quad r=v w
$$

subject to the relation $p q=r^{2}$. The original curve is given by an equation $F(p, t)=0$ and we are really looking for is functions $q(p, t)$ and $r(p, t)$ subject to the relation $p q(p, t)=$ $r(p, t)^{2}$. In addition, if $p \rightarrow \infty$ and $t \sim p^{N_{c}+1}$, then $r(p, t) \sim \mu p, q(p, t) \sim \mu^{2} p$, whereas in the other two asymptotic regions as $p \rightarrow \infty$ we require $q(p, t)$ to remain finite. Thus, we see that $q(p, t)$ is a function on the compactified curve with a single pole. The existence of such a function requires that the compactified curve is a $\mathbf{C P}^{1}$. More precisely, there are two possibilities. Either the compactified curve is a $\mathbf{C P}^{1}$, or the compactified curve consists of several components, and the component containing the point corresponding to the leftmost NS fivebrane is a $\mathbf{C P}^{1}$. We call these two possibilities the non-factorized and factorized cases respectively, and discuss them separately.

non-factorized curve. In this case, the entire curve must be a $\mathrm{CP}^{1}$, and the fact that $q(p, t)$ is a function with a single pole on this $\mathbf{C P}^{1}$ implies that $q$ is a good global coordinate on the entire curve. We should therefore be able to express $p$ and $t$ in terms of $q$. At three points on the $\mathrm{CP}^{1} p$ becomes infinite and is a good local coordinate there; one of these three points is $q=\infty$, the other correspond to finite values of $q$, and $p$ is therefore given by

$$
p=\frac{\left(q-q_{1}\right)\left(q-q_{2}\right)\left(q-q_{3}\right)}{\mu^{2}\left(q-q_{4}\right)\left(q-q_{5}\right)} .
$$

Because $\left\{q_{1}, q_{2}, q_{3}\right\} \cap\left\{q_{4}, q_{5}\right\}=\emptyset, r(q)=\sqrt{p(q) q}$ always has a branch cut and is never globally well-defined. We conclude that the rotation is impossible.

factorized curve. It is straightforward to see that $F(t, v)$ can be factored in terms of two polynomials in at most two ways. Taking $\Lambda_{N=2, S p}=\Lambda_{N=2, S O}=1$ for simplicity these are

1) $\quad F(t, v)=(t+d)\left(t^{2}-\left(v^{2} P_{N_{c}}\left(v^{2}\right)+c+d\right) t-d^{-1} v^{2}\right)$,

2) $\quad F(t, v)=\left(t+d v^{2}\right)\left(t^{2}-\left(v^{2} P_{N_{c}}\left(v^{2}\right)+c+d v^{2}\right) t-d^{-1}\right)$.

The first case requires $Q_{N_{f}}\left(v^{2}\right)=-d^{-1} v^{2}-d\left(v^{2} P_{N_{c}}\left(v^{2}\right)+c+d\right)$, so that $N_{f}=N_{c}+1$. In addition, according to (5.11), $c^{2}=4 Q(0)=-4 d(c+d)$, so that $c=-2 d$. The first factor in $F(t, v)$ corresponds to the middle NS fivebrane, which we do not want to change ${ }^{1}$.

\footnotetext{
${ }^{1}$ From the type IIA point of view, this factorization corresponds to sticking 2 of the $2 N_{f}$ D4 branes
} 
The second factor in $F(t, v)$ can not be further factorized and must therefore be a $\mathbf{C P}^{1}$. Applying the same logic as in the non-factorized case to this piece of the curve we find that $p=v^{2}$ must be of the form $\left(q-q_{1}\right)\left(q-q_{2}\right) / \mu^{2}\left(q-q_{3}\right)$. In order for $r=\sqrt{p(q) q}$ to be well-defined we need $q_{1}=q_{2}$ and $q_{3}=0$. But this implies that there is only one value of $q$ for which $p=0$, which contradicts the fact that there are two distinct values of $t$ for $p=0$, namely $t=0$ and $t=-d$ ( $d=0$ is clearly impossible). Hence, the curve cannot be rotated.

It remains to analyze the second possibility in (5.14). That one requires $Q_{N_{f}}\left(v^{2}\right)=$ $-d^{-1}-d v^{2}\left(v^{2} P_{N_{c}}\left(v^{2}\right)+c+d v^{2}\right)$, fixing $N_{f}=N_{c}+2$. Furthermore, $c^{2}=4 Q(0)=-4 d^{-1}$. Again, the first factor in (5.14) corresponds to the middle NS fivebrane, so that we have to require that the second factor corresponds to a $\mathrm{CP}^{1}$. The second factor describes the curve of a pure $S p\left(N_{c}\right)$ gauge theory, and we already know from section 3 when this curve can be rotated. There are precisely $N_{c}+1$ discrete possibilities for the parameters in $P_{N_{c}}\left(v^{2}\right)$, corresponding to the $N_{c}+1$ discrete vacua of pure $S p\left(N_{c}\right)$ gauge theory. In addition there are two choices for $c$, so that the total number of vacua that survives the mass perturbation is $2\left(N_{c}+1\right)$.

Comparing the results of the field theory with those of the brane analysis, we see that there is a complete agreement.

\subsection{Runaway Behavior}

We have seen that the structure of the spaces of supersymmetric vacua obtained from the brane and from field theory agree. It is an interesting question whether the runaway behavior of the scalar potential that we discussed for $N_{f}=N_{c}+1$ can be seen in the brane language as well. A priori this is a more difficult question. In field theory, it involves knowledge of the Kähler potential. As was demonstrated in [43], it is very difficult to obtain quantitative information about the Kähler potential using the Mtheory fivebrane in the eleven dimensional supergravity limit of M theory. In the present case we do not need to know the detailed quantitative structure of the Kähler potential, we are only interested in some qualitative features, and one may hope that the brane will still reproduce these qualitative features.

Let us first try to rephrase runaway behavior in the brane language. To find a brane configuration for a given theory we first need to specify some boundary conditions. In the

in the $\mathrm{O} 4$ plane so that the charge of the $\mathrm{O} 4$ plane to the left and right of the middle NS fivebrane is equal. The remaining D4 branes to the left and right of the middle NS fivebrane can then be connected and subsequently one can move the middle NS fivebrane away from the configuration. 
case we have been considering so far, namely an $N=2$ gauge theory with gauge group $S p\left(N_{c}\right) \times S O\left(2 N_{f}\right)$ broken to $N=1$ by a mass term for $\Phi_{\text {Sp }}$, these boundary conditions are that as $v \rightarrow \infty$, there are three distinct asymptotic regions: (i) $t \sim v^{2 N_{c}+2}, w \sim \mu v$, (ii) $t \sim v^{2 N_{f}-2 N_{c}-2}, w \rightarrow 0$ and (iii) $t \sim v^{2-2 N_{f}}, w \rightarrow 0$. In addition there are certain conditions that have to do with the M-theory O5 plane. As we discussed previously, there should be no $t$-configuration, i.e. there should be no transversal intersection of a single fivebrane with the orientifold plane, the orientifold plane should have the right fivebrane charge and the brane configuration should be $\mathbf{Z}_{2}$ invariant. We will ignore the subtleties associated to the orientifold plane for the time being and first consider the case without an orientifold plane. Consider a given brane configuration that satisfies the right boundary conditions and which has four trivial directions corresponding to the four dimensions where the field theory lives. Such a brane configuration corresponds to a particular field configuration in the field theory. If the brane corresponds to a supersymmetric cycle, it describes a supersymmetric field configuration. From the fivebrane action it is clear that the area of the fivebrane corresponds to the scalar potential of the field theory, and a minimal area fivebrane configuration corresponds to a minimum of the scalar potential. Therefore runaway behavior should manifest itself in the brane picture in the following way: given a brane configuration that satisfies the right boundary conditions one should always be able to deform it so as to reduce its area, without ever reaching a limiting configuration. If one keeps on reducing its area some of the parameters describing the brane configuration will runaway to infinity.

Let us now try to apply this picture to the case with $N_{f}=N_{c}+1$, where we expect such runaway behavior from field theory.

We first consider some general features of minimal surfaces in Kähler manifolds with metric $G_{i \bar{\jmath}}$, where $X^{i}$ are the holomorphic coordinates in target space. A surface is locally given by some functions $X^{i}(z, \bar{z})$ depending on the complex coordinates $z, \bar{z}$. The area of a surface is reparametrization invariant and we will fix this reparametrization invariance by the Virasoro conditions

$$
G_{i \bar{\jmath}} \partial X^{i} \partial X^{\bar{\jmath}}=G_{i \bar{\jmath}} \bar{\partial} X^{i} \bar{\partial} X^{\bar{\jmath}}=0 .
$$

This has the advantage, familiar from string theory, that the area of the surface is given by the simple expression

$$
S=\int_{\Sigma} d^{2} z G_{i \bar{\jmath}}\left(\partial X^{i} \bar{\partial} X^{\bar{\jmath}}+\bar{\partial} X^{i} \partial X^{\bar{\jmath}}\right) .
$$

As shown in section 4.3 , this can be rewritten as

$$
S=2 \int_{\Sigma} d^{2} z G_{i \bar{\jmath}} \bar{\partial} X^{i} \partial X^{\bar{\jmath}}+\int_{\Sigma} X^{*} \omega
$$


where $\omega$ is the Kähler form of target space. We also showed that in order to find a minimal surface in a given homology class and with certain given boundary conditions, we can consider the second term in (5.17) to be constant and drop it. We are thus left with the task of minimizing the regularized volume

$$
S=\operatorname{Area}^{\prime}(\Sigma)=2 \int_{\Sigma} d^{2} z G_{i \bar{\jmath}} \bar{\partial} X^{i} \partial X^{\bar{\jmath}} .
$$

As we explained, an advantage of this expression is that in the case where we are dealing with holomorphic boundary conditions this expression is finite (which is in some sense the definition of holomorphic boundary conditions). It also clearly shows that any holomorphic surface is automatically minimal. The variation of $X^{i}$ in (5.18) leads to the minimal surface equation

$$
G_{i \bar{\jmath}} \partial \bar{\partial} X^{\bar{\jmath}}+\bar{\partial} X^{\bar{k}} \partial X^{\bar{\jmath}} \partial_{\bar{k}} G_{i \bar{\jmath}}=0 .
$$

Let us now discuss two specific situations. First consider the case where $G_{i \bar{\jmath}}$ is constant. The minimal surface equation reads $\partial \bar{\partial} X^{i}=\partial \bar{\partial} X^{\bar{\imath}}=0$. This implies that $X^{i}=X^{i}(z)+X^{i}(\bar{z})$, where $X^{i}(z)$ is a possibly multi-valued holomorphic function. Because (5.18) is finite, $X^{i}(\bar{z})$ must be bounded as one approaches infinity. There are no bounded non-trivial anti-holomorphic functions on a Riemann surface, even if one allows for multi-valuedness. Therefore $X^{i}(\bar{z})$ must be identically zero and the minimal surface must necessarily be holomorphic. An alternative way to see this is to partially integrate (5.18) and then to use the minimal surface condition to show it vanishes. Because (5.18) is positive definite and vanishes only for holomorphic curves, the minimal surface must be holomorphic.

The second situation we want to consider is what happens when we have a holomorphic surface with given boundary conditions and then we change the holomorphic boundary conditions, like turning on a mass $\mu$ for $\Phi_{\mathrm{Sp}}$. At infinity, the change in the boundary conditions is very large. However, this is a change of holomorphic boundary conditions only, so that we expect a large change in the holomorphic part of $X^{i}$ and the antiholomorphic part of $X^{\bar{j}}$, but only a very small change in the anti-holomorphic part of $X^{i}$ and the holomorphic part of $X^{\bar{j}}$. Since (5.18) only involves the latter, we can construct a minimal surface for small $\mu$ as a small perturbation of the one for $\mu=0$. Indeed, if we substitute $X^{i} \rightarrow X^{i}+\xi^{i}, X^{\bar{\jmath}} \rightarrow X^{\bar{\jmath}}+\xi^{\bar{\jmath}}$ in (5.19) and expand to first order in $\xi$ using the fact that $X^{i}$ is holomorphic and $X^{\bar{j}}$ is anti-holomorphic we get

$$
G_{i \bar{\jmath}} \partial \bar{\partial} \xi^{\bar{\jmath}}+\bar{\partial} X^{\bar{k}} \partial \xi^{\bar{\jmath}} \partial_{\bar{k}} G_{i \bar{\jmath}}=0
$$

which is only an equation for the very small quantity $\partial \xi^{\bar{j}}$. We can rewrite (5.20) as 
$\bar{\partial}\left(G_{i \bar{\jmath}} \partial \xi^{\bar{\jmath}}\right)=0$ which implies that

$$
\int d^{2} z G_{i \bar{\jmath}} \bar{\partial} \xi^{i} \partial \xi^{\bar{\jmath}}=0
$$

and therefore $\xi^{i}$ has to be holomorphic. We conclude that for sufficiently small $\mu$ any minimal surface must necessarily be holomorphic.

This statement could in principle get modified in the presence of an orientifold, due to the additional potential energy it creates. However, as long as there are no holomorpic $t$-configurations after the perturbation, we believe we can restrict our attention to non-t-configurations only. The discussion above then still applies in the presence of an orientifold, as long as the distance between the fivebrane and the $\mathbf{Z}_{2}$ fixed plane is much larger than the eleven-dimensional Planck length. In particular, in the presence of an orientifold we cannot exclude the existence of a local nonzero minimum of the scalar potential in the region where the eleven dimensional supergravity approximation breaks down. This is similar to the situation in field theory, where one cannot say anything about the behavior of the scalar potential at strong coupling due to lack of knowledge of the Kähler potential in that region.

We therefore see that for $N_{f}=N_{c}+1$ that for small enough $\mu$ (i) there is no holomorphic surface satisfying the right boundary conditions and (ii) that any minimal surface has to be holomorphic. The only way to match these two observations is if (5.18) can be made arbitrarily small, but cannot be made equal to zero. A simple analogy of this situation is the problem to find the shortest real curve in the $x, y$-plane that approaches $y=a$ as $x \rightarrow-\infty$, and approaches $y=b$ as $x \rightarrow+\infty$. Any curve that satisfies the right boundary conditions is clearly not the straight line. We can always make the curve straighter and straighter, thereby reducing its length, but we never reach the point where it becomes a straight line.

Exactly the same situation appears in the case at hand. Given some surface, we can always reduce its area, for instance by replacing the surface inside a large sphere by the minimal surface inside the sphere, keeping the boundary conditions on the sphere fixed. We can make the sphere arbitrarily large, but never reach an exact minimal surface.

It is now clear that this behavior is precisely the manifestation of runaway behavior in the brane picture. It also shows that under quite general circumstances (e.g. in the case of the product group for all $N_{f} \neq N_{c}+2$ ) where supersymmetry is dynamically broken, we do expect runaway behavior in the scalar potential. Our discussion of the case where $G_{i \bar{\jmath}}$ is constant implies that similar things happen in the case of products of $S U$ gauge groups. Thus, runaway behavior in the presence of holomorphic boundary conditions 
seems to be a quite general phenomenon, except in situations with additional interactions as discussed in section 4. Finding counterexamples where a non-holomorphic minimal surface satisfies holomorphic boundary conditions is obviously an important problem, for which something quite non-trivial in the brane picture must happen.

\section{Acknowledgement}

We thank Hitoshi Murayama for many useful discussions. We furthermore would like to acknowledge discussions with Amit Giveon, David Kutasov, Joe Lykken, Al Shapere and Scott Thomas. We thank Savas Dimopoulos and Michael Peskin for remarks and questions that lead to an improvement of the paper.

This work is supported in part by NSF grant PHY-951497 and DOE grant DE-AC0376SF00098. JdB is a fellow of the Miller Institute for Basic Research in Science. 


\section{References}

[1] A. Klemm, W. Lerche, P. Mayr, C.Vafa, N. Warner, "Self-Dual Strings and N=2 Supersymmetric Field Theory," hep-th/9604034, Nucl.Phys. B477 (1996) 746-766.

[2] A. Hanany and E. Witten, "Type IIB Superstrings, BPS Monopoles, And ThreeDimensional Gauge Dynamics," hep-th/9611230, Nucl.Phys. B492 (1997) 152.

[3] S. Elitzur, A. Giveon, D. Kutasov, "Branes and N=1 Duality in String Theory," hepth/9702014, Phys.Lett. B400 (1997) 269.

[4] H. Ooguri and C. Vafa, "Geometry of $N=1$ Dualities in Four-Dimensions," hepth/9702180, Nucl. Phys. B500 (1997) 62.

[5] J.L.F. Barbon, "Rotated Branes and N=1 Duality," hep-th/9703051, Phys.Lett. B402 (1997) 59-63.

[6] E. Witten, "Solutions of Four-Dimensional Field Theories via $M$ Theory," hep-th/9703166, Nucl.Phys. B500 (1997) 3.

[7] N. Evans, C.V. Johnson and A.D. Shapere, "Orientifolds, Branes, and Duality of 4D Gauge Theories," hep-th/9703210, Nucl.Phys. B505 (1997) 251.

[8] J.H. Brodie and A. Hanany, "Type IIA Superstrings, Chiral Symmetry and $N=14-\mathrm{D}$ Gauge Theory Dualities", hep-th/9704043, Nucl. Phys. B506 (1997) 157.

[9] A. Brandhuber, J. Sonnenschein, S. Theisen and S. Yankielowicz, "Brane Configurations and 4-D Field Theory Dualities", hep-th/9704044, Nucl. Phys. B502 (1997) 125.

[10] C. Ahn and K. Oh, "Geometry, D-Branes and $N=1$ Duality in Four Dimensions.1.", hep-th/9704061, Phys. Lett. B412 (1997) 274.

[11] S. Elitzur, A. Giveon, D. Kutasov, E. Rabinovici, A. Schwimmer, "Brane Dynamics and N=1 Supersymmetric Gauge Theory," hep-th/9704104, Nucl.Phys. B505 (1997) 202-250.

[12] R. Tatar, "Dualities in 4-D Theories with Product Gauge Groups from Brane Configurations", hep-th/9704198.

[13] C. Ahn, "Geometry, D-Branes and $N=1$ Duality in Four Dimensions.2.", hep-th/9705004.

[14] C. Ahn and R. Tatar, "Geometry, D-Branes and $N=1$ Duality in Four Dimensions with Product Gauge Group", hep-th/9705106.

[15] K. Landsteiner, E. Lopez and D.A.. Lowe, ' $N=2$ Supersymmetric Gauge Theories, Branes and Orientifolds', hep-th/9705199, Nucl. Phys. B507 (1997) 197.

[16] A. Brandhuber, J. Sonnenschein, S. Theisen, S. Yankielowicz, "M Theory And SeibergWitten Curves: Orthogonal and Symplectic Groups," hep-th/9705232, Nucl.Phys. B504 (1997) 175.

[17] A. Hanany and A. Zaffaroni, "Chiral Symmetry from Type IIA Branes", hep-th/9706047. 
[18] K. Hori, H. Ooguri and Y. Oz, "Strong Coupling Dynamics of Four-Dimensional N=1 Gauge Theories from M Theory Fivebrane," hep-th/9706082, Adv. in Theor. and Math. Phys. 1 (1997) 1.

[19] E. Witten, "Branes And The Dynamics Of QCD", hep-th/9706109, Nucl.Phys. B507 (1997) 658-690.

[20] J. Furukawa, "S Duality in $N=2$ QCD and Nonabelian Duality in $N=1$ QCD with M-Theory", hep-th/9706121.

[21] A. Brandhuber, N. Itzhaki, V. Kaplunovsky, J. Sonnenschein and S. Yankielowicz, "Comments on the M Theory Approach to $N=1$ SQCD and Brane Dynamics," hep-th/9706127, Phys. Lett. B410 (1997) 27.

[22] C. Ahn, K. Oh and R. Tatar, "Branes, Geometry and $N=1$ Duality with Product Gauge Groups of SO and SP", hep-th/9707027.

[23] A. Gorsky, S. Gukov and A. Mironov, "Multiscale $N=2$ SUSY Field Theories, Integrable Systems and Their Stringy/Brane Origin.1," hep-th/9707120"

[24] A. Hanany, M. Strassler and A. Zaffaroni, "Confinement and Strings in MQCD", hepth/9707244.

[25] S. Nam, K. Oh and S.-J. Sin, "Superpotentials of $N=1$ Supersymmetric Gauge Theories from M Theory", hep-th/9707247.

[26] M. Henningson and P. Yi "Four-dimensional BPS-spectra via M-theory," hep-th/9707251.

[27] T. Nakatsu, K. Ohta, T. Yokono and Y. Yoshida, "Higgs Branch of N=2 SQCD and M theory Branes," hep-th/9707258; T. Nakatsu, K. Ohta and T. Yokono, "On the Baryonic Branch Root of N=2 MQCD," hep-th/9712029.

[28] M. Schmaltz and R. Sundrum, "N = 1 Field Theory Duality from M-theory," hepth/9708015.

[29] J. Barbon and A. Pasquinucci, "D0-Branes, Constrained Instantons and $D=4$ Super Yang-Mills Theories," hep-th/9708041.

[30] J. de Boer and Y. Oz, "Monopole Condensation and Confining Phase of $N=1$ Gauge Theories Via M Theory Fivebrane," hep-th/9708044.

[31] K. Landsteiner and E. Lopez, "New Curves from Branes," hep-th/9708118.

[32] A. Mikhailov, "BPS States and Minimal Surfaces," hep-th/9708068.

[33] C. Csaki and W. Skiba, "Duality in Sp and SO Gauge Groups from M Theory," hepth/9708082.

[34] N. Evans, M. Schwetz, "The Field Theory of Nonsupersymmetric Brane Configurations," hep-th/9708122. 
[35] C. Ahn, K. Oh and R. Tatar, "Sp$\left(N_{c}\right)$ Gauge Theories and M Theory Fivebrane," hepth/9708127.

[36] A. Giveon and O. Pelc, 'M Theory, Type IIA String and 4D $N=1 \mathrm{SUSY} S U\left(N_{L}\right) \otimes S U\left(N_{R}\right)$ Gauge Theory', hep-th/9708168.

[37] J. Lykken, E. Poppitz and S.P. Trivedi, "Chiral Gauge Theories from D-Branes," hepth/9708134; "M(ore) on Chiral Gauge Theories from D-Branes," hep-th/9712193.

[38] C. Ahn, K. Oh and R. Tatar, "M Theory Fivebrane Interpretation for Strong Coupling Dynamics of $S O\left(N_{c}\right)$ Gauge Theories," hep-th/9709096; "M Theory Fivebrane and Confining Phase of $\mathrm{N}=1 S O\left(N_{c}\right)$ Gauge Theories," hep-th/9712005; C. Ahn, "Confining Phase of $\mathrm{N}=1 S p\left(N_{c}\right)$ Gauge Theories via M Theory Fivebrane," hep-th/9712149.

[39] A. Volovich, "Domain Wall in MQCD and Supersymmetric Cycles in Exceptional Holonomy Manifolds," hep-th/9710120.

[40] J.L.F. Barbon and A. Pasquinucci, "Softly Broken MQCD and the Theta Angle," hepth/9711030.

[41] A. Fayyazuddin and M. Spalinski, "The Seiberg-Witten Differential From M-Theory," hepth/9706087; "Extended Objects in MQCD," hep-th/9711083.

[42] P.S. Howe, N.D. Lambert and P.C. West, "Classical M-Fivebrane Dynamics and Quantum N=2 Yang-Mills," hep-th/9710034; N.D. Lambert and P.C. West, "Gauge Fields and MFivebrane Dynamics,", hep-th/9712040.

[43] J. de Boer, K. Hori, H. Ooguri and Y. Oz, 'Kähler Potential and Higher Derivative Operators from M Theory Fivebrane', hep-th/9711143.

[44] S. Terashima, "Supersymmetric Gauge Theories with Classical Groups via M Theory Fivebrane," hep-th/9712172.

[45] K. Landsteiner, E. Lopez and D.A. Lowe, "Duality of Chiral $N=1$ Supersymmetric Gauge Theories via Branes," hep-th/9801002.

[46] I. Brunner, A. Hanany, A. Karch and D. Lüst, "Brane Dynamics and Chiral Nonchiral Transitions," hep-th/9801017.

[47] S. Elitzur, A. Giveon, D. Kutasov and D. Tsabar, "Branes, Orientifolds and Chiral Gauge Theories", hep-th/9801020.

[48] S.-J. Sin, "The Super-potential and Holomorphic properties of the MQCD", hepth/9801032.

[49] K.-I. Izawa and T. Yanagida, "Dynamical Supersymmetry Breaking in Vector-like Gauge Theories," hep-th/9602180, Prog.Theor.Phys. 95 (1996) 829-830.

[50] K. Intriligator and S. Thomas, "Dynamical Supersymmetry Breaking on Quantum Moduli Spaces," hep-th/9603158, Nucl.Phys. B473 (1996) 121-142. 
[51] N. Seiberg, "Exact Results on the Space of Vacua of Four Dimensional SUSY Gauge Theories," hep-th/9402044, Phys. Rev. D49 (1994) 6857-6863.

[52] K. Intriligator and P. Pouliot, "Exact Superpotentials, Quantum Vacua and Duality in Supersymmetric $S P\left(N_{c}\right)$ Gauge Theories," hep-th/9505006, Phys.Lett. B353 (1995) 471476.

[53] N. Arkani-Hamed and H. Murayama "Renormalization Group Invariance of Exact Results in Supersymmetric Gauge Theories," hep-th/9705189.

[54] P.C. Argyres and A.D. Shapere, "The Vacuum Structure of N=2 SuperQCD with Classical Gauge Groups," hep-th/9509175, Nucl.Phys. B461 (1996) 437-459.

[55] P.C. Argyres, M.R. Plesser and A.D. Shapere, "N=2 Moduli Spaces and N=1 Dualities for $S O\left(n_{c}\right)$ and $U S p\left(2 n_{c}\right)$ SuperQCD," hep-th/9608129, Nucl.Phys. B483 (1997) 172-186.

[56] K. Intriligator and N. Seiberg, "Lectures on supersymmetric gauge theories and electricmagnetic duality," hep-th/9509066, Nucl.Phys.Proc.Suppl. 45BC (1996) 1-28.

[57] J. Polchinski, "TASI Lectures on D-Branes," hep-th/9611050.

[58] E. Witten, "Five-branes And $M$-Theory On An Orbifold," hep-th/9512219, Nucl.Phys. B463 (1996) 383-397.

[59] K. Dasgupta and S. Mukhi, "Orbifolds of M-theory," hep-th/9512196, Nucl.Phys. B465 (1996) 399-412.

[60] N. Seiberg, "IR Dynamics on Branes and Space-Time Geometry," hep-th/9606017, Phys.Lett. B384 (1996) 81.

[61] K. Hori, H. Ooguri and C. Vafa, "Non-Abelian Conifold Transitions and N=4 Dualities in Three Dimensions," hep-th/9705220, Nucl.Phys. B504 (1997) 147-174.

[62] K. Becker, M. Becker and A. Strominger. "Fivebranes, Membranes and Non-Perturbative String Theory," hep-th/9507158.

[63] M. Bershadsky, V. Sadov and C. Vafa, "D-Branes and Topological Field Theories." hepth/9511222.

[64] K.Becker, M.Becker, D.R.Morrison, H.Ooguri, Y.Oz and Z.Yin, "Supersymmetric Cycles in Exceptional Holonomy Manifolds and Calabi-Yau 4-Folds," hep-th/9608116.

[65] A. Hanany and K. Hori, "Branes and $N=2$ Theories in Two Dimensions," hep-th/9707192.

[66] N. Seiberg, 'Electric-Magnetic Duality in Supersymmetric Non-Abelian Gauge Theories', hep-th/9411149.

[67] K. Intriligator and N. Seiberg, 'Duality, Monopoles, Dyons, Confinement and Oblique Confinement in Supersymmetric $S O\left(N_{c}\right)$ Gauge Theories', hep-th/9503179.

[68] K. Hori, to appear. 
[69] S. Kachru and E. Silverstein, "Singularities, Gauge Dynamics, and Nonperturbative Superpotentials in String Theory," hep-th/9608194, Nucl.Phys. B482 (1996) 92-104. 